\title{
Liquid sloshing in an upright circular tank under periodic and transient excitations
}

\author{
Liang, Hui; Santo, Harrif; Shao, Yanlin; Law, Yun Zhi; Chan, Eng Soon
}

Published in:

Physical Review Fluids

Link to article, DOI:

10.1103/PhysRevFluids.5.084801

Publication date:

2020

Document Version

Publisher's PDF, also known as Version of record

Link back to DTU Orbit

Citation (APA):

Liang, H., Santo, H., Shao, Y., Law, Y. Z., \& Chan, E. S. (2020). Liquid sloshing in an upright circular tank under periodic and transient excitations. Physical Review Fluids, 5(8), [084801].

https://doi.org/10.1103/PhysRevFluids.5.084801

\section{General rights}

Copyright and moral rights for the publications made accessible in the public portal are retained by the authors and/or other copyright owners and it is a condition of accessing publications that users recognise and abide by the legal requirements associated with these rights.

- Users may download and print one copy of any publication from the public portal for the purpose of private study or research.

- You may not further distribute the material or use it for any profit-making activity or commercial gain

- You may freely distribute the URL identifying the publication in the public portal

If you believe that this document breaches copyright please contact us providing details, and we will remove access to the work immediately and investigate your claim. 


\title{
Liquid sloshing in an upright circular tank under periodic and transient excitations
}

\author{
Hui Liang $\odot,{ }^{1, *}$ Harrif Santo, ${ }^{1}$ Yanlin Shao $\odot,{ }^{2,3}$ Yun Zhi Law, ${ }^{1}$ and Eng Soon Chan ${ }^{1,4}$ \\ ${ }^{1}$ Technology Centre for Offshore and Marine, Singapore (TCOMS), Singapore 118411 \\ ${ }^{2}$ Department of Mechanical Engineering, Technical University of Denmark, Lyngby 2800, Denmark \\ ${ }^{3}$ College of Shipbuilding Engineering, Harbin Engineering University, Harbin 150001, China \\ ${ }^{4}$ Department of Civil and Environmental Engineering, National University of Singapore, \\ Singapore 117576, Singapore
}

(Received 26 March 2020; accepted 8 July 2020; published 3 August 2020)

In this paper, the liquid sloshing problem in an upright circular tank undergoing an oscillation in a single degree of freedom is considered. A fully nonlinear time-domain harmonic polynomial cell (HPC) method based on overset mesh is developed to delve into the flow physics, and the comparison is made with the weakly nonlinear multimodal theory. Both time-harmonic (or periodic) and transient-type oscillations are considered. For the time-harmonic oscillation, planar and swirling waves (including time-harmonic and periodically modulated swirling waves) occur when the excitation frequency is close to the lowest natural frequency. The swirling direction is dependent on initial conditions. The periodically modulated swirling waves switch back and forth between swirling waves and planar waves. The occurrence of swirling waves results in lateral hydrodynamic force and roll moment on top of in-line force and pitch moment components acting on the tank. The sloshing response in terms of surface elevations contains higher harmonic components, while the hydrodynamic forces and moments on the tank are remarkably linear. A NewWave type of excitation is imposed which represents on average the most probable maximum excitation to be expected for a given sea state, and narrow-banded sloshing responses are observed. This allows application of a designer-wave type of excitation which would excite the most probable maximum response in sloshing. A focused-wave-type excitation is also considered, and an impulselike feature is observed in the in-line force and pitch moment components. The total in-line force and pitch moment are further decomposed into inertial component, which is induced by the acceleration of the external motion, and residual component, which is closely associated with the liquid sloshing. It is found that under a focused-wave-type excitation the inertial component contribution is more dominant, while under a designer-wave-type excitation the residual component dominates instead.

DOI: 10.1103/PhysRevFluids.5.084801

\section{INTRODUCTION}

Liquid sloshing representing liquid motion in a storage tank with a free surface is a fundamental problem. The sloshing in an upright circular tank is relevant for ship motions and floating storage facilities due to water waves in marine hydrodynamics [1], closed fish tanks in aquaculture [2], on-land storage tanks subjected to earthquake excitation in civil engineering [3], and rocket fuel problems in aeronautic engineering [4]. The presence of sloshing flow has a strong influence on

*liang_hui@tcoms.sg 
the motion and response behaviors of structures, and thus it is of both theoretical and practical importance. The comprehensive theoretical basis of the sloshing as well as its related problems have been presented in Refs. [5,6].

The study of liquid sloshing in an upright circular tank can be traced back to the 1960s by Hutton [7]. In his experiments, the tank is undergoing a time-harmonic translational oscillation, and the observation shows that both planar and nonplanar sloshing waves are existent. Miles [8,9] developed weakly nonlinear four-dimensional modal equations in the steady state to quantitatively investigate responses near the lowest natural frequency. Four kinds of responses, which are (1) planar harmonic, (2) swirling harmonic, (3) periodically modulated sinusoid, and (4) chaotically modulated sinusoid, are predicted. The experimental studies by Funakoshi and Inoue [10] and Royon-Lebeaud et al. [11] lend strong support to Miles' theoretical analysis and found that swirling waves have a hard-spring behavior. Faltinsen and Timokha [6] developed a weakly nonlinear multimodal theory in the time domain based on the Bateman-Luke variational formulation, which is more general compared to Miles' work [9], enabling the computation of hydrodynamic force and the incorporation of transient effects. In the weakly nonlinear multimodal theory, the first five natural modes are nonlinearly coupled. However, it is not limited to only five modes, as the higher modes can be included through linear modal equations. Besides the excitation of single degree of freedom, attention has also been paid to the mean mass transport of liquid in the tank induced by a rotary oscillation off resonance [12-15].

In the present study, the focus is placed on the sloshing flow inside of an upright circular tank subjected to surging motion. For the occurrence of swirling sloshing waves in the steady state, it takes long time to build up, and nonlinearity must be accounted for. Therefore, a time efficient, fully nonlinear solver is required. This is important particularly since most of the existing studies focus on the use of periodic excitation. However, transient-type excitation associated with an extreme event has seldom been considered in the literature, and therefore little is known about the underlying physics.

In marine and offshore engineering, periodic excitation on the sloshing flow is used to represent regular waves. For applications in deeper water, periodic waves are not representative of an extreme wave and therefore compact transient wave groups are widely used instead to represent an extreme wave event. For linear random waves with Gaussian surface elevation statistics, the average shape of an extreme wave in time tends to a scaled autocorrelation of the underlying wave spectrum $[16,17]$. In marine and offshore engineering, such compact wave groups became known as a NewWave [18]. A comparison was made between the shape of the measured large linearized wave crests and the shape predicted by the NewWave model for measured time series in the North Sea [19], in the Gulf of Mexico [20], and recently in a shallower water [21]. The good agreement justifies the use of the NewWave as a reasonable model for the linear part of large ocean wave crests. Apart from NewWave excitation, an impulsive focused-wave-type excitation is also considered, representing an extreme or freak wave type of event. Focused wave groups are widely used as an input excitation to analyze the response of marine and offshore systems, e.g., see Refs. [22-25].

For these purposes, a fully nonlinear analysis of the near resonance liquid sloshing in an upright circular tank subjected to surging motion is carried out. An overset-mesh-based, fully nonlinear harmonic polynomial cell (HPC) method in the time domain is then developed to delve into the underlying flow physics. Both periodic and transient (and/or impulsive) excitations are considered. A comparative study is made with the weakly nonlinear multimodal theory by Faltinsen and Timokha [6].

The HPC method proposed by Shao and Faltinsen [26] is extensively used to investigate nonlinear water waves as well as wave-structure interactions. The application of high-order polynomials and the resultant sparse coefficient matrix indicate that the HPC method is time efficient and of high accuracy. Subsequent work and improvements were conducted in recent years [27-30] to circumvent more challenging problems. In contrast to the boundary element method (BEM), which is widely applied in potential flow computation, the HPC method is more time efficient and the computational cost is roughly proportional to the number of unknowns. On the other hand, the conventional BEM involves quadratic memory usage and operations even though an efficiently 


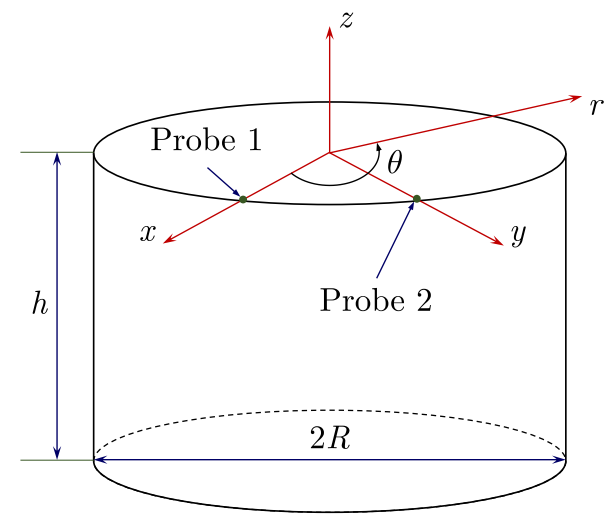

FIG. 1. Definition of dimension and coordinate system of an upright circular tank.

iterative solver is used [31]. Moreover, the numerical test in [26] indicates that the HPC method requires much less computational time to achieve the same level of accuracy compared to the BEM.

Nevertheless, a two-dimensional study by Ma et al. [29] shows that the accuracy is drastically reduced when the aspect ratio of the cell deviates from unity or the cell is significantly distorted. In the case of liquid sloshing in an upright circular tank, however, stretching or distortion of cells is inevitable when the boundary-fitted grid is used. Therefore, various strategies, such as use of an immersed boundary, overset mesh, multigrid, etc., are suggested to be incorporated into the HPC method [30]. In light of that, a fully nonlinear HPC method incorporating the overset-mesh technique is developed herein.

The layout of the present paper is as follows. The basic equations together with the linear and weakly nonlinear modal theories are set forth in Sec. II. In Sec. III, a fully nonlinear time-domain HPC method incorporating the overset-mesh technique is developed, and the accuracy on the Neumann-type boundaries is improved by introducing ghost nodes outside of the computational domain. By using the developed numerical model, the flow physics of liquid sloshing in an upright circular tank is investigated in Secs. IV and V. When the tank is undergoing time-harmonic oscillation with a single degree of freedom near the lowest natural frequency, swirling waves are observed, which include time-harmonic swirling waves and periodically modulated swirling waves caused by mode interactions, resulting in lateral force and roll moment on the tank. The swirling behavior is not dependent on initial conditions, but initial conditions can determine the swirling direction. When an impulsive NewWave-type excitation is imposed, no swirling waves were observed in both fully nonlinear HPC results and weakly nonlinear multimodal analysis. The induced sloshing waves will last a long time in the tank because of the absence of radiation damping; nevertheless, the concept of designer-wave-type excitation can be usefully applied to derive the largest response. When a more impulsive focused-wave-type excitation is imposed, an impulselike feature is observed in the hydrodynamic force and moment acting on the tank. Swirling waves are not observed under the transient-type excitation. Finally, concluding remarks and future perspectives are presented in Sec. VI.

\section{ANALYTICAL ASPECTS OF LIQUID SLOSHING IN AN UPRIGHT CIRCULAR TANK}

It is assumed that the fluid is incompressible and inviscid, and the flow is irrotational so that there exists a velocity potential $\Phi$ with its gradient defined as velocity components $\nabla \Phi=$ $(U, V, W)=\vec{U}$. Consider an upright circular tank with liquid inside. A noninertial tank-fixed cylindrical coordinate system $\operatorname{Or} \theta z$ is defined with the $\operatorname{Or} \theta$ plane on the undisturbed free surface and the $O z$ axis coinciding with cylindrical axis and pointing positively upwards, as shown in Fig. 1. 
The radius of the tank is $R$, and the water depth is $h$. Here, only the surging motion is considered. More general formulations are referred to Ref. [6].

\section{A. Boundary-value problem}

For the problem of liquid sloshing in an upright circular tank, the fluid domain $\Omega$ is bounded by a tank surface $S$ (consisting of a bottom and a sidewall) and a free surface $F$. The tank is in the surging motion $\eta_{1}$ in $x$ direction. The boundary-value problem is defined as follows [6]:

$$
\begin{aligned}
\frac{\partial^{2} \Phi}{\partial z^{2}}+\frac{1}{r} \frac{\partial}{\partial r}\left(r \frac{\partial \Phi}{\partial r}\right)+\frac{1}{r^{2}} \frac{\partial^{2} \Phi}{\partial \theta^{2}} & =0 \quad \text { in } \Omega, \\
\frac{\partial \Phi}{\partial n} & =\dot{\eta}_{1} n_{x} \quad \text { on } \quad S, \\
\frac{\partial \Phi}{\partial t}+\mu \Phi+\frac{1}{2}|\nabla \Phi|^{2}-\dot{\eta}_{1} \frac{\partial \Phi}{\partial x}+g z & =0 \quad \text { on } \quad F, \\
\frac{\partial \Phi}{\partial n} & =\dot{\eta}_{1} n_{x}-\frac{\partial Z / \partial t}{|\nabla Z|} \text { on } F,
\end{aligned}
$$

where $n_{x}$ is the $x$ component of the normal vector, $Z(x, y, z, t)=z-\zeta(x, y, t)=0$ is the freesurface equation with $\zeta(x, y, t)$ defined as the free-surface elevation, and $\mu$ is an artificial viscosity coefficient. Here, the Rayleigh-type viscous damping coefficient $\mu$ is incorporated in the dynamic free-surface boundary condition to damp out the transient effect. For a smooth tank without internal structures which may dissipate energy, the damping for sloshing response is normally very small, which is mainly contributed by the boundary layer on the tank surface and the fluid bulk [6]. To avoid unphysical results at resonance frequencies, a damping coefficient as small as $0.5 \%$ of the critical damping of the first natural modes will be applied, i.e., $\mu=\varepsilon \times 2 \omega_{1,1}$ with $\varepsilon=0.5 \%$. Similar damping ratios have been applied in Faltinsen et al. [32].

\section{B. Natural mode}

Natural modes are nontrivial solutions to the linear boundary-value problem with zero tank excitation, and they are the foundation of the modal theory which will be set forth later. Solving the boundary-value problem (1) via the separation of variables gives rise to the following natural modes [6]:

$$
\begin{aligned}
& \varphi_{m, n, 1}(r, \theta, z)=\frac{\cosh \left[k_{m, n}(z+h)\right]}{\cosh \left(k_{m, n} h\right)} \frac{J_{m}\left(k_{m, n} r\right)}{J_{m}\left(k_{m, n} R\right)} \cos (m \theta), \\
& \varphi_{m, n, 2}(r, \theta, z)=\frac{\cosh \left[k_{m, n}(z+h)\right]}{\cosh \left(k_{m, n} h\right)} \frac{J_{m}\left(k_{m, n} r\right)}{J_{m}\left(k_{m, n} R\right)} \sin (m \theta),
\end{aligned}
$$

where $m=0,1,2, \ldots, n=1,2,3, \ldots$, and $k_{m, n}$ satisfies

$$
J_{m}^{\prime}\left(k_{m, n} R\right)=J_{m}^{\prime}\left(\iota_{m, n}\right)=0,
$$

with $J_{m}(\cdot)$ defined as the Bessel function of the first kind and order $m$ [33]. Given $k_{m, n}$, the eigenvalues $\kappa_{m, n}$ and natural frequencies $\omega_{m, n}$ can be determined:

$$
\kappa_{m, n}=\frac{\omega_{m, n}^{2}}{g}=k_{m, n} \tanh \left(k_{m, n} h\right) .
$$

\section{Linear modal theory}

When the boundary-value problem is linearized, the linear modal theory can be applied. Here we reformulate the linear modal equations accounting for viscous damping effects in the sense 
of Rayleigh. In the linear modal theory, the free-surface elevation $\zeta$ and velocity potential $\Phi$ are expanded as [6]

$$
\begin{gathered}
\zeta(r, \theta, t)=\sum_{m=0}^{\infty} \sum_{n=0}^{\infty} \sum_{j=1}^{2} \beta_{m, n, j}(t) \varphi_{m, n, j}(r, \theta, 0), \\
\Phi(r, \theta, z, t)=\dot{\eta}_{1} x+\sum_{m=0}^{\infty} \sum_{n=0}^{\infty} \sum_{j=1}^{2} R_{m, n, j}(t) \varphi_{m, n, j}(r, \theta, z),
\end{gathered}
$$

with $m+n>0$. By substituting expansions (5a) and (5b) into the kinematic and dynamic freesurface boundary conditions (1d) and (1c) and omitting nonlinear terms, a set of linear ordinary differential equations can be obtained:

$$
\ddot{\beta}_{m, n, j}+2 \varepsilon \omega_{m, n} \dot{\beta}_{m, n, j}+\omega_{m, n}^{2} \beta_{m, n, j}=K_{m, n, j},
$$

where the forcing term is written as

$$
K_{m, n, j}=-\frac{\lambda_{1(m, n, j)}}{\chi_{m, n, j}}\left(\ddot{\eta}_{1}+\mu \dot{\eta}_{1}\right)
$$

with nonzero coefficients given by [6]

$$
\begin{aligned}
\chi_{1, n, 1} & =\chi_{1, n, 2}=\frac{\rho \pi R^{3}\left(\iota_{1, n}^{2}-1\right)}{2 \iota_{1, n}^{3} \tanh \left(\iota_{1, n} h / R\right)}, \\
\lambda_{1(1, n, 1)} & =\lambda_{1(1, n, 2)}=\frac{\rho \pi R^{3}}{\iota_{1, n}^{2}} .
\end{aligned}
$$

\section{Weakly nonlinear multimodal theory}

In the weakly nonlinear single-dominant multimodal theory [6,32], the lowest five modes along with their mutual interactions are considered. According to Moiseev ordering, five modal functions with lowest natural frequencies are written as

$$
\begin{aligned}
& p_{1}=\beta_{1,1,1} \sim \epsilon^{1 / 3}, \\
& r_{1}=\beta_{1,1,2} \sim \epsilon^{1 / 3}, \\
& p_{0}=\beta_{0,1,1} \sim \epsilon^{2 / 3}, \\
& p_{2}=\beta_{2,1,1} \sim \epsilon^{2 / 3}, \\
& r_{2}=\beta_{2,1,2} \sim \epsilon^{2 / 3},
\end{aligned}
$$

where $\epsilon$ is a nondimensional parameter associated with the smallness of the excitation. Here, higher than third-order modes are neglected. According to [6], the five-dimensional nonlinear modal system coupling first- and second-order modes is written as

$$
\begin{aligned}
\ddot{p}_{1} & +2 \varepsilon \omega_{1,1} \dot{p}_{1}+\omega_{1,1}^{2} p_{1} \\
& +K_{1} p_{1}\left(p_{1} \ddot{p}_{1}+\dot{p}_{1}^{2}+r_{1} \ddot{r}_{1}+\dot{r}_{1}^{2}\right) \\
& +K_{2}\left(r_{1}^{2} \ddot{p}_{1}+2 r_{1} \dot{r}_{1} \dot{p}_{1}-r_{1} p_{1} \ddot{p}_{1}-2 p_{1} \dot{r}_{1}^{2}\right) \\
& +K_{3}\left(p_{2} \ddot{p}_{1}+r_{2} \ddot{r}_{1}+\dot{r}_{1} \dot{r}_{2}+\dot{p}_{1} \dot{p}_{2}\right) \\
& -K_{4}\left(p_{1} \ddot{p}_{2}+r_{1} \ddot{r}_{2}\right)
\end{aligned}
$$




$$
\begin{aligned}
& +K_{5}\left(p_{0} \ddot{p}_{1}+\dot{p}_{1} \dot{p}_{0}\right)+K_{6} p_{1} \ddot{p}_{0} \\
= & -\frac{\left.\lambda_{1(1,1,1}\right)}{\chi_{1,1,1} R}\left(\ddot{p}_{1}+\mu \dot{\eta}_{1}\right), \\
\ddot{r}_{1} & +2 \varepsilon \omega_{1,1} \dot{r}_{1}+\omega_{1,1}^{2} r_{1} \\
& +K_{1} r_{1}\left(r_{1} \ddot{r}_{1}+\dot{r}_{1}^{2}+p_{1} \ddot{p}_{1}+\dot{p}_{1}^{2}\right) \\
& +K_{2}\left(p_{1}^{2} \ddot{r}_{1}+2 p_{1} \dot{r}_{1} \dot{p}_{1}-r_{1} p_{1} \ddot{p}_{1}-2 r_{1} \dot{p}_{1}^{2}\right) \\
& -K_{3}\left(p_{2} \ddot{r}_{1}-r_{2} \ddot{p}_{1}+\dot{r}_{1} \dot{p}_{2}-\dot{p}_{1} \dot{r}_{2}\right) \\
& +K_{4}\left(p_{1} \ddot{p}_{2}-p_{1} \ddot{r}_{2}\right) \\
& +K_{5}\left(p_{0} \ddot{r}_{1}+\dot{r}_{1} \dot{p}_{0}\right)+K_{6} r_{1} \ddot{p}_{0}=0, \\
\ddot{p}_{0} & +2 \varepsilon \omega_{0,1} \dot{p}_{0}+\omega_{0,1}^{2} p_{0} \\
& +K_{10}\left(r_{1} \ddot{r}_{1}+p_{1} \ddot{p}_{1}\right)+K_{8}\left(\dot{r}_{1}^{2}+\dot{p}_{1}^{2}\right)=0, \\
\ddot{p}_{2} & +2 \varepsilon \omega_{2,1} \dot{p}_{2}+\omega_{2,1}^{2} p_{2} \\
& +K_{9}\left(r_{1} \ddot{r}_{1}-p_{1} \ddot{p}_{1}\right)+K_{7}\left(\dot{r}_{1}^{2}-\dot{p}_{1}^{2}\right)=0, \\
\ddot{r}_{2} & +2 \varepsilon \omega_{2,1} \dot{r}_{2}+\omega_{2,1}^{2} r_{2} \\
& -K_{9}\left(r_{1} \ddot{p}_{1}+p_{1} \ddot{r}_{1}\right)-2 K_{7} \dot{r}_{1} \dot{p}_{1}=0,
\end{aligned}
$$

where $K_{j}$ with $j=1,2, \ldots, 10$ are tabulated in [6]. It is possible to account for higher modes, which can be incorporated in modal equations analogous to the linear modal theory in Sec. II C.

The applicability of the multimodal theory requires that the first natural mode is predominant and the assumptions in Eq. (7) hold. If so, the multimodal theory is highly efficient and accurate. Nevertheless, it is essentially a weakly nonlinear method based on the perturbation scheme. When there is strong nonlinearity, the first mode is not necessarily predominant, and therefore a higher-order model is required. Alternatively, a fully nonlinear numerical approach, which will be introduced in the next section, can be used.

\section{AN OVERSET-MESH-BASED HPC METHOD FOR LIQUID SLOSHING IN AN UPRIGHT CIRCULAR TANK}

The fully nonlinear time-domain HPC method based on the overset mesh is developed herein to investigate the liquid sloshing problem in an upright circular tank.

\section{A. Summary of the HPC method}

A three-dimensional Cartesian coordinate system $O x y z$ is defined with the $O x y$ plane coinciding with the undisturbed free-surface and $O z$ axis orienting positively upwards. The fluid domain is discretized into overlapping hexahedral cells, and each stencil cell is defined as the combination of neighboring hexahedral cells with 26 grid points as illustrated in Fig. 2. The velocity potential within each cell is represented as a linear combination of $N$ harmonic polynomials:

$$
\Phi(X, Y, Z)=\sum_{j=1}^{N} b_{j} P_{j}(X, Y, Z),
$$

where $P_{j}(X, Y, Z)$ with $j=1,2, \ldots, N$ mean harmonic polynomials associated with Legendre polynomials in a spherical coordinate system [26]. Here, $X, Y$, and $Z$ are local coordinates relative to the stencil center. Due to the fact that harmonic polynomials satisfy the Laplace equation naturally, 


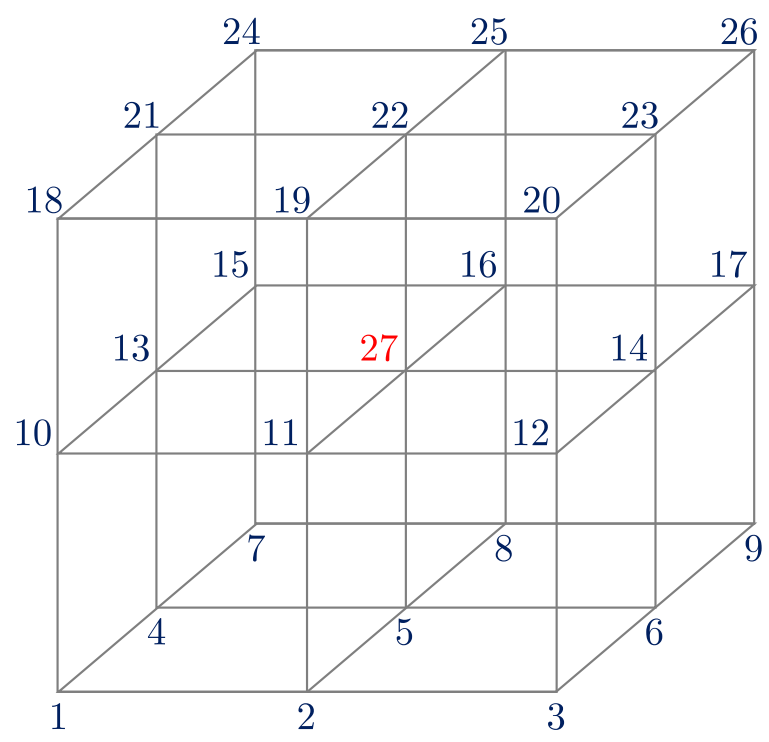

FIG. 2. A hexahedral cell with 27 nodes with the 27 th node defined as the stencil center.

there is no need to impose the Laplace equation. By imposing (9) on the 26 stencil points, one can obtain a linear equation system in the form of

$$
[\boldsymbol{A}] \cdot\{\boldsymbol{b}\}=\{\boldsymbol{\Phi}\}
$$

with elements $A_{i, j}$ given by

$$
A_{i, j}=P_{j}\left(X_{i}, Y_{i}, Z_{i}\right), \quad i=1,2, \ldots, 26 .
$$

Here, $N$ is not necessarily equal to 26 . When $N<26$, the least-squares fitting can be used. By taking the inverse of (10), we can obtain the vector $\{\boldsymbol{b}\}$ :

$$
b_{j}=\sum_{i=1}^{26} c_{j, i} \Phi_{i} \quad \text { with } \quad j=1, \ldots, N,
$$

where $c_{j, i}$ are elements of the matrix $[\boldsymbol{A}]^{-1}$ or $\left[[\boldsymbol{A}]^{T} \cdot[\boldsymbol{A}]\right]^{-1} \cdot[\boldsymbol{A}]^{T}$. Substituting (12) into (9) gives rise to

$$
\begin{aligned}
\Phi(X, Y, Z) & =\sum_{j=1}^{N}\left[\sum_{i=1}^{26} c_{j, i} \Phi_{i}\right] P_{j}(X, Y, Z) \\
& =\sum_{i=1}^{26}\left[\sum_{j=1}^{N} c_{j, i} P_{j}(X, Y, Z)\right] \Phi_{i} .
\end{aligned}
$$

Equation (13) indicates that the velocity potential at any point in the cell can be interpolated by the velocity potential on the surrounding nodes of the cell. By rendering $X=0, Y=0$, and $Z=0$, one can obtain the continuity equation at the stencil center:

$$
\Phi_{27}=\sum_{i=1}^{26} V_{i} \Phi_{i} \quad \text { with } \quad V_{i}=c_{1, i} .
$$




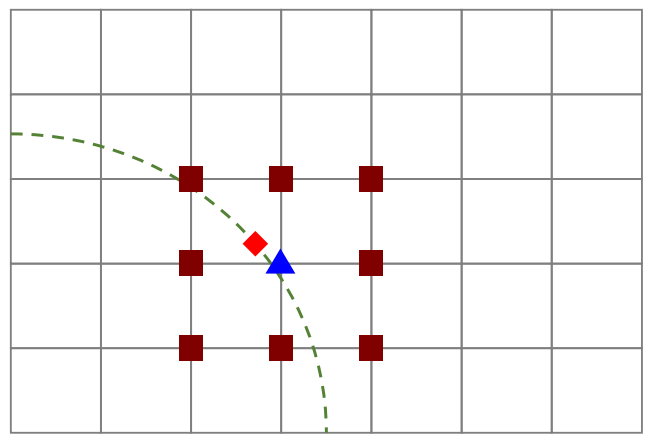

$\checkmark$ Receptor node

$\Delta$ Nearest node

- Stencil nodes

FIG. 3. Interpolation of the node on the fringe of a circular grid system.

Due to the fact that the harmonic polynomial set adopted in the three-dimensional HPC method includes all third-order terms as well as incomplete fourth-, fifth- and sixth-order terms, the HPC method is higher than third-order accuracy.

On the solid boundaries, the Neumann-type boundary condition requiring the derivative of the potential is implemented by directly taking the derivative of harmonic polynomials:

$$
\nabla \Phi(X, Y, Z)=\sum_{i=1}^{26} U_{i} \Phi_{i}
$$

with

$$
U_{i}=\sum_{j=1}^{N} c_{j, i} \nabla P_{j}(X, Y, Z) .
$$

In a polar coordinate system, an Euler rotation with respect to the azimuth angle of the stencil center is performed.

\section{B. Overset-mesh technique}

Due to the fact that increasing the aspect ratio of a cell results in larger errors [29], an oversetmesh-based HPC method is developed here. As shown in Fig. 3, the overset mesh consists of a body-fitted mesh (referred to as foreground mesh) and a background mesh (represented by solid lines) overlapping each other. At the point in the fluid domain and surrounded by other nodes, the Laplace equation is imposed as usual. At the point at the fringe (plotted by a dashed line), however, the information is determined via polynomial interpolation based on the physical quantities on the other set of mesh.

As described in Fig. 3, when the node at the fringe of the foreground mesh (represented by a diamond) is identified (receptor node), the nearest node in the background mesh is first searched. Then the stencil nodes surrounding the searched node can be found, and these stencil nodes are also referred to as donor nodes (denoted by squares). Finally, the velocity potential at the receptor node is interpolated by the information at stencil nodes and is expressed as

$$
\Phi^{\text {receptor }}=\sum_{j=1}^{26} c_{j} \Phi_{j}^{\text {stencil }},
$$

where $c_{j}$ is the solution to the linear equation system

$$
[A]\{\boldsymbol{c}\}=\{1,0, \ldots, 0\}^{T},
$$




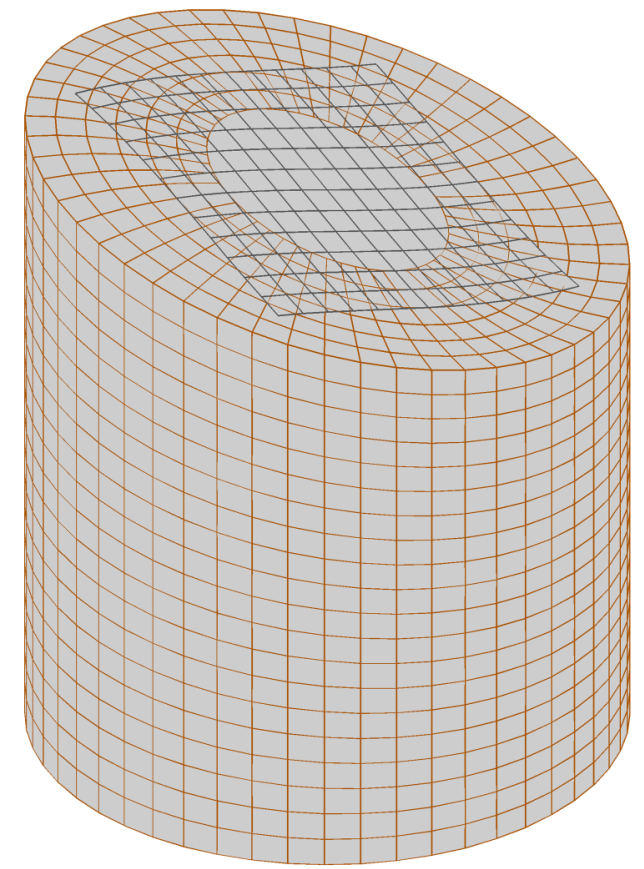

FIG. 4. The overset mesh in the upright circular tank.

with

$$
A_{i, j}=P_{j}\left(x_{i}-x_{0}, y_{i}-y_{0}, z_{i}-z_{0}\right),
$$

where $\left(x_{0}, y_{0}, z_{0}\right)$ is the coordinate of the receptor node, and $\left(x_{i}, y_{i}, z_{i}\right)$ are the coordinates of stencil nodes. In this manner, the relation between the receptor node in the foreground mesh and the stencil (donor) nodes in the background mesh is set up, and the Laplace equation is also satisfied naturally at the receptor node. On the free surface where the Dirichlet-type boundary condition is satisfied in the time-domain simulation, the potential at the receptor node is interpolated by biquadratic polynomials based on the donor nodes in the background mesh.

\section{Liquid sloshing in an upright circular tank}

The fully nonlinear time-domain analysis of the liquid sloshing problem in an upright circular tank is carried out in a noninertial frame of reference fixed to the tank. The overset mesh is illustrated in Fig. 4. To improve the accuracy on the vertical sidewall, the ghost node technique is adopted. When the boundary-fitted grid is used, computational nodes are distributed along boundaries and inside of the computational domain. The node at which the boundary condition is satisfied is off-centered due to the fact that a centered stencil will reach beyond the computational domain. When the fictitious ghost nodes are introduced outside of the computational domain as illustrated in Fig. 5, the boundaries are immersed and thus the node on boundaries is centered. To make sure the system is determinate, both the governing equation and tank-wall boundary condition are enforced at the boundary nodes [34]. In this scenario, the boundary condition is satisfied at the stencil center, leading to significant improvements in the accuracy on the boundaries.

On the intersection between the free surface and sidewall, the free-surface and tank-wall boundary conditions are imposed. On the free surface $F$, the physical information is updated via 


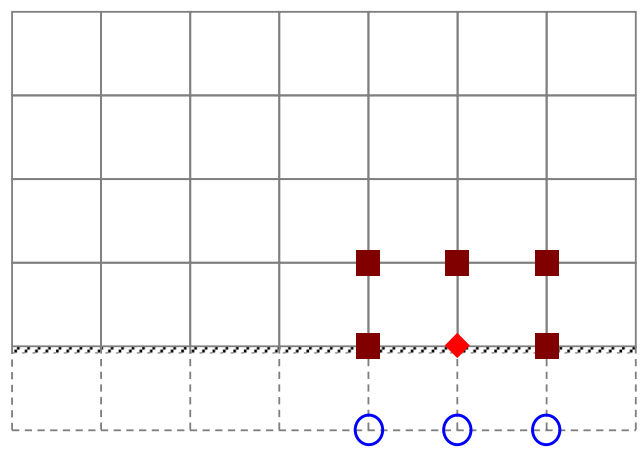

Stencil center

Ghost nodes

Stencil nodes

Solid boundary

FIG. 5. Schematic of ghost nodes outside of the computational domain.

the semi-Lagrangian description, which considers a point moving vertically, and the kinematic and dynamic free-surface boundary conditions are written as

$$
\begin{aligned}
\frac{d^{s} \zeta}{d t} & =\frac{\partial \Phi}{\partial z}-\frac{\partial \Phi}{\partial x} \frac{\partial \zeta}{\partial x}-\frac{\partial \Phi}{\partial y} \frac{\partial \zeta}{\partial y}+\dot{\eta}_{1} \frac{\partial \zeta}{\partial x} \\
\frac{d^{s} \Phi}{d t} & =-\frac{1}{2}|\nabla \Phi|^{2}+\dot{\eta}_{1} \frac{\partial \Phi}{\partial x}+g z+\frac{\partial \Phi}{\partial z} \frac{d^{s} \zeta}{d t}-\mu \Phi,
\end{aligned}
$$

on $z=\zeta(x, y, t)$.

In order to invoke swirling waves, an initial disturbance of the free-surface elevation, written as

$$
\zeta(r, \theta, t=0)=0.05 \frac{J_{1}\left(k_{1,1} r\right)}{J_{1}\left(k_{1,1} R\right)} \sin \theta,
$$

is prescribed, where $k_{1,1}$ is defined in Eq. (3). In the numerical examples considered in Secs. IV and $\mathrm{V}$, Eq. (21) is used as the initial condition.

In the numerical implementation, the explicit fourth-order Runge-Kutta scheme is used to integrate the boundary conditions (20a) and (20b) to update the elevation $\zeta$ and velocity potential $\Phi$ on the free surface at each time step. To ensure numerical stability, a hybrid numerical filter combining a noncentered filter [35] and the centered 13-point, 10th-order Savitzky-Golay filter [36] is used to remove possible sawtooth waves.

To compute the hydrodynamic force and moment experienced by the tank, Bernoulli's equation in the noninertial coordinate system fixed to the tank given in [6] is used, and the force and moment acting on the tank are written as

$$
\left\{\begin{array}{l}
\vec{F} \\
\vec{M}
\end{array}\right\}=-\rho \iint_{S}\left(\frac{\partial \Phi}{\partial t}+\frac{1}{2}|\nabla \Phi|^{2}-\dot{\eta}_{1} \frac{\partial \Phi}{\partial x}\right)\left\{\begin{array}{c}
\vec{n} \\
\vec{s} \times \vec{n}
\end{array}\right\} d S,
$$

where $\vec{F}$ and $\vec{M}$ denote the force and moment vectors, respectively, and $\vec{s}$ is the vector from the reference point to the point on the tank surface. In the present study, the reference point is defined at the origin $(0,0,0)$, and $\vec{n}$ points positively outwards the fluid domain. In the following context, the force $\vec{F}$ and moment $\vec{M}$ by the tank is nondimensionalized by $\vec{f}=\vec{F} /\left(\rho g R^{3}\right)$ and $\vec{m}=\vec{M} /\left(\rho g R^{4}\right)$, respectively.

\section{PERIODIC EXCITATION}

A periodic excitation is considered in this section. Here we consider a cylindrical tank with radius $R=1 \mathrm{~m}$ and liquid depth $h=2 \mathrm{~m}$ as shown in Fig. 1. In this case, the lowest natural frequency determined by formulation (4) is $\omega_{1,1} \approx 4.2465 \mathrm{rad} \mathrm{s}^{-1}$. The tank undergoes the surge motion (in $x$ 

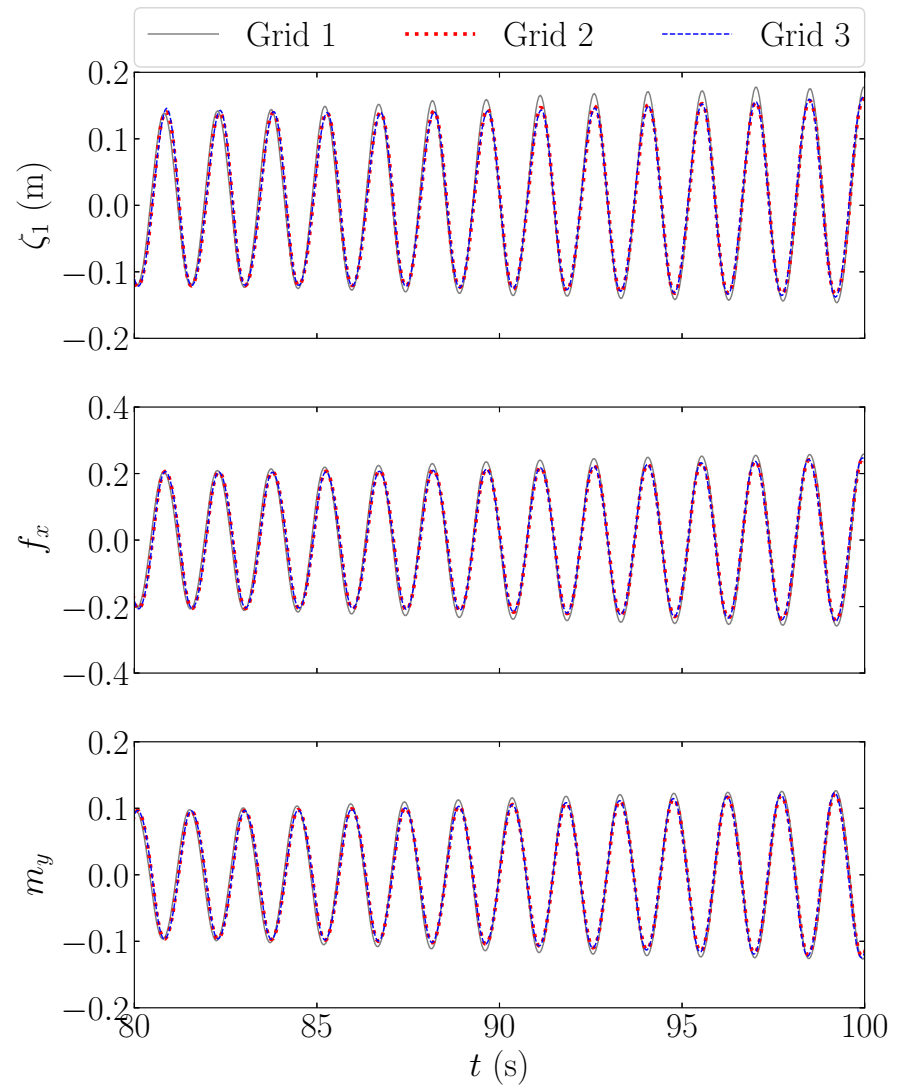

FIG. 6. Time histories of the free-surface elevation $\zeta_{1}$ (top), in-line force $f_{x}$ (middle), and pitch moment $m_{y}$ (bottom) for three different grid resolutions at $\omega=\omega_{1,1}$.

direction) in the form of

$$
\eta_{1}=0.0024 R \sin (\omega t)
$$

where $\omega$ is the angular frequency of the surge motion.

\section{A. Convergence test}

To test the convergence of the grid, time histories of the free-surface elevation $\zeta_{1}$, in-line force $f_{x}$, and pitch moment $m_{y}$ at $\omega=\omega_{1,1}$ are exhibited in the top, middle, and bottom subplots in Fig. 6 . Three different grid resolutions are considered, which correspond to 5049, 11 613, and 35743 grid points, and are identified as "Grid 1," "Grid 2," and "Grid 3," respectively. It is observed that the results associated with Grid 1 slightly deviates from the other two groups of results, whereas the results with Grid 2 and Grid 3 are in good agreement, indicating that both Grid 2 and Grid 3 could yield converged results. In the numerical examples hereafter, Grid 2 as illustrated in Fig. 4 is adopted.

\section{B. Wave pattern, force, and moment}

Figure 7 presents the time history of a periodic type of excitation (top row) for $\omega=0.98 \omega_{1,1}$, as well as the resulting responses in terms of the surface elevation at two probe locations (second row), and the normalized hydrodynamic forces and moments on the tank (third and bottom rows) for the 


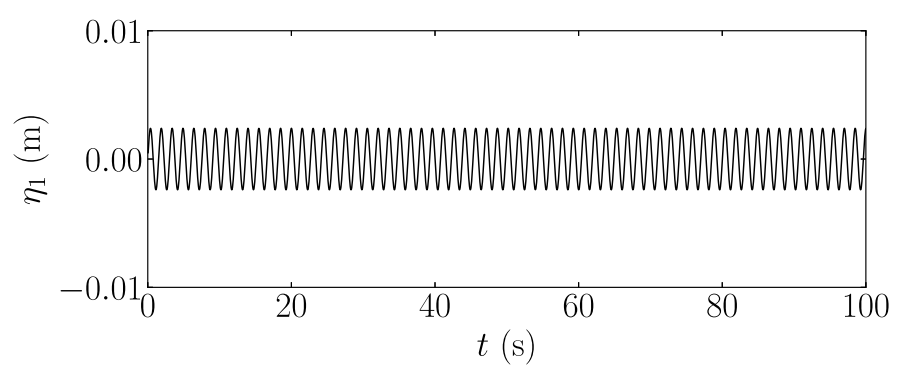

(a)

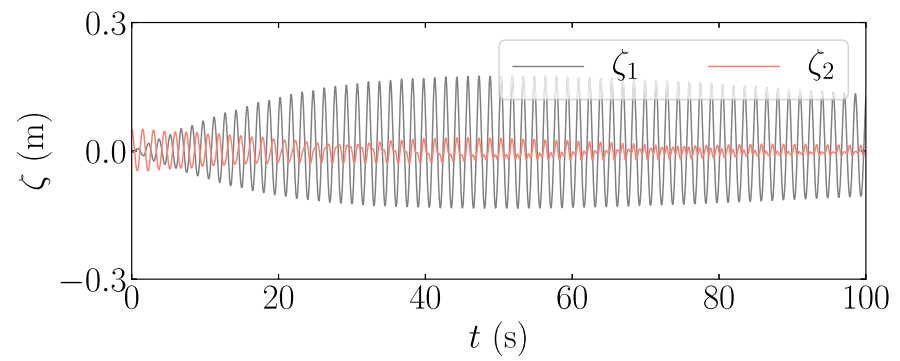

(b)

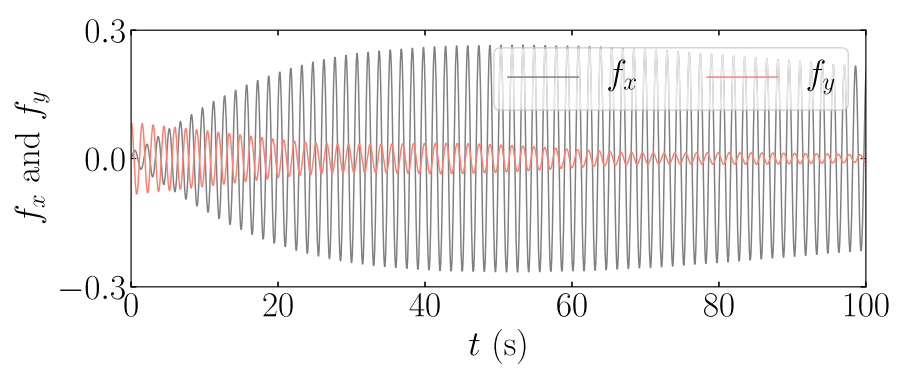

(c)

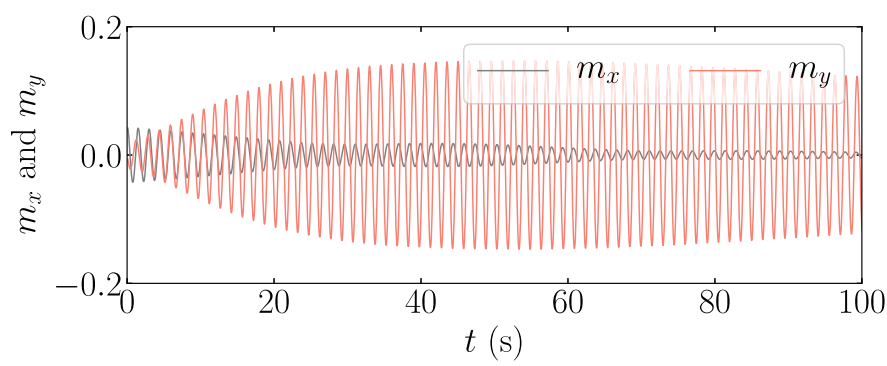

(d)

FIG. 7. Time history of a periodic type of excitation (a) and the resulting responses in terms of surface elevation (b), normalized hydrodynamic force (c) and moment (d) on the tank determined by the fully nonlinear HPC method for $\omega=0.98 \omega_{1,1}$.

in-line $\left(f_{x}\right)$ and lateral $\left(f_{y}\right)$ force components as well as roll $\left(m_{x}\right)$ and pitch $\left(m_{y}\right)$ moment components. In this numerical example, $\zeta_{1}, f_{x}$, and $m_{y}$ exhibit a buildup of responses before reaching the steady state at $t>50 \mathrm{~s}$, while $\zeta_{2}$ gradually decays to zero after being perturbed at $t=0 \mathrm{~s}$. It is worth noting 

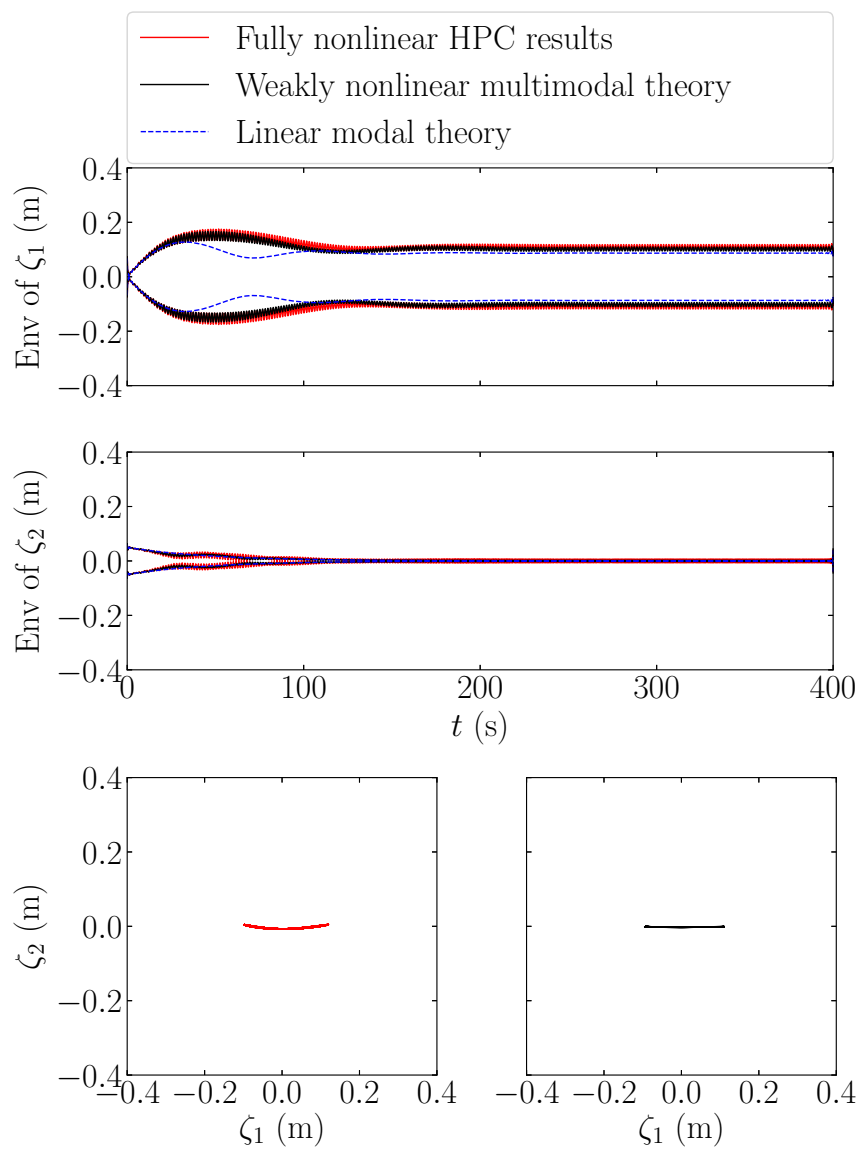

FIG. 8. The top and middle panels show the time histories of the envelopes of $\zeta_{1}$ and $\zeta_{2}$, respectively. The present fully nonlinear HPC results (red) are in comparison with the results obtained from the weakly nonlinear multimodal theory (black) and the linear modal theory (blue). The bottom panel displays the phase plot defined as the trajectory of $\zeta_{1}$ and $\zeta_{2}$ from $t=200 \mathrm{~s}$ to $t=400 \mathrm{~s}$ obtained from HPC (left) and the multimodal theory (right). All results are for $\omega=0.98 \omega_{1,1}$.

that the surface elevation is more weakly nonlinear (thus containing higher harmonic contribution), while the hydrodynamic force and moment are remarkably linear.

We present results from five different excitation frequencies ranging between $\omega=0.98 \omega_{1,1}$ and $\omega=1.02 \omega_{1,1}$. Figures 8-12 show the results in terms of the envelopes of $\zeta_{1}$ and $\zeta_{2}$ in the first two subplots, while the bottom subplot shows the results in terms of phase plot, defined as the trajectory of $\zeta_{1}$ and $\zeta_{2}$ in the time interval from $t=200 \mathrm{~s}$ to $t=400 \mathrm{~s}$. The comparison is made with the results determined by the linear modal theory, as well as the weakly nonlinear multimodal theory elucidated in Sec. II.

For periodic excitations at $\omega=0.98 \omega_{1,1}, \omega=1.01 \omega_{1,1}$, and $\omega=1.02 \omega_{1,1}, \zeta_{2}$ gradually decays to zero while $\zeta_{1}$ reaches a steady state after $t=200 \mathrm{~s}$. It can be observed that the fully nonlinear HPC results are in good agreement with the results obtained from the weakly nonlinear multimodal theory, whereas the difference from the results determined by the linear modal theory is observed. In these three cases, sloshing waves propagate forth and back due to vanishing $\zeta_{2}$, thus exhibiting planar wave behavior.

When the surging frequency is $\omega=0.99 \omega_{1,1}$ as shown in Fig. 9, both $\zeta_{1}$ and $\zeta_{2}$ reach the steady state after $t=200 \mathrm{~s}$ but exhibit periodically modulated swirling behaviors. In this scenario, $\zeta_{1}$ and $\zeta_{2}$ 

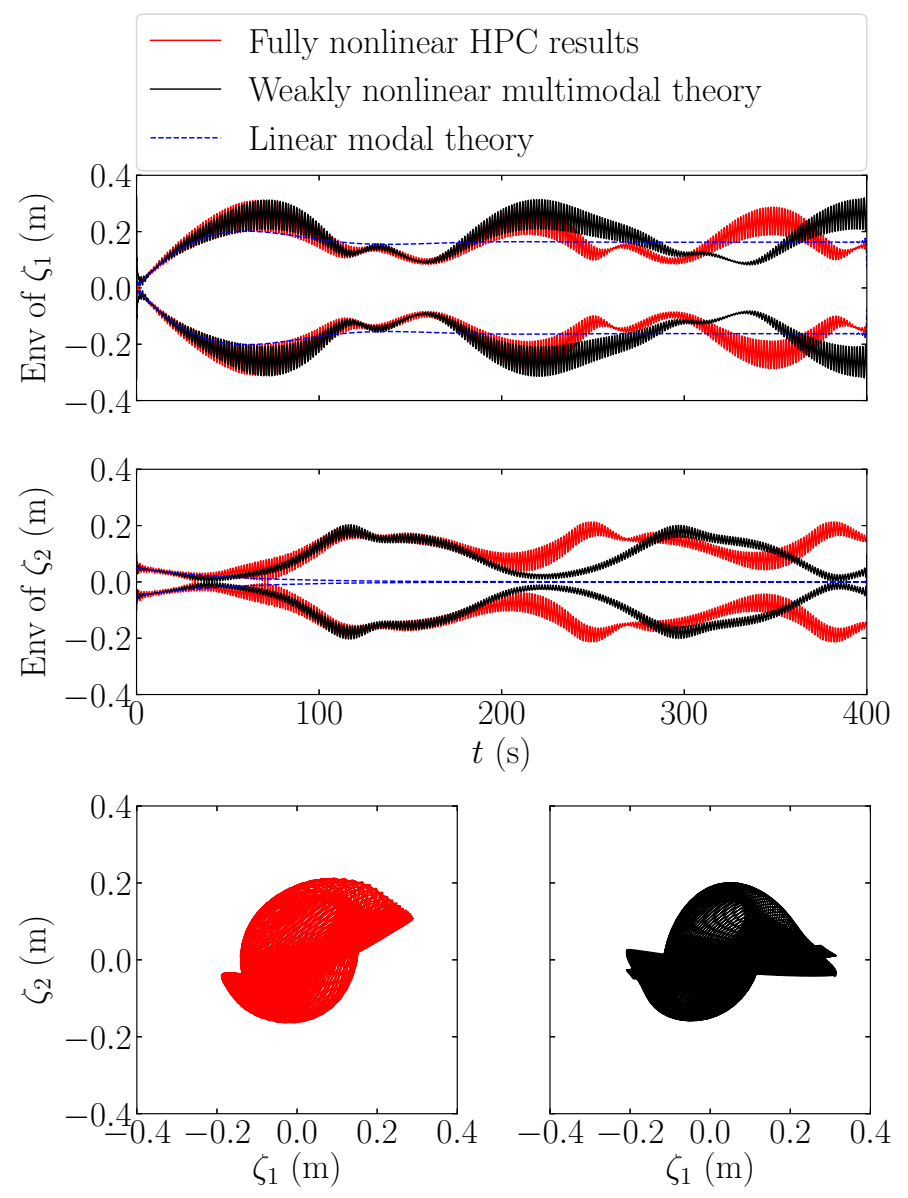

FIG. 9. Same as Fig. 8 but for $\omega=0.99 \omega_{1,1}$.

are not always $\pi / 2$ apart. The phase plot is no longer a closed circle representing irregular behaviors. Both the fully nonlinear HPC method and the weakly nonlinear multimodal theory are capable of capturing the periodically modulated behaviors, whereas the results obtained from the linear modal theory exhibit planar behavior, at odds with the other two groups of results. By observing the envelopes of $\zeta_{1}$ and $\zeta_{2}$, the amplitude of $\zeta_{2}$ increases while that of $\zeta_{1}$ decreases, and vice versa. Therefore, there is energy exchange between the longitudinal symmetric mode $\varphi_{1,1,1}$ and transverse symmetric mode $\varphi_{1,1,2}$ defined in Eq. (2). However, an appreciable difference between the results determined by the fully nonlinear HPC method and the weakly nonlinear multimodal theory is observed. Further analysis of periodically modulated swirling waves is described in Sec. IV C.

When the surging frequency coincides with the lowest natural frequency, i.e., at $\omega=\omega_{1,1}$ as shown in Fig. 10, $\zeta_{1}$ and $\zeta_{2}$ reach the steady state after $t=200 \mathrm{~s}$, indicating that the sloshing waves in the tank propagate both longitudinally and transversely. Moreover, both $\zeta_{1}$ and $\zeta_{2}$ have a phase difference of nearly $\pi / 2$ as shown in Fig. 13, thus exhibiting swirling wave behavior. The phase plot in the form of a closed circle corroborates the swirling behavior. Both envelope and time history in Figs. 10 and 13 demonstrate that the fully nonlinear HPC results agree well with the results determined by the weakly nonlinear multimodal theory in terms of both amplitude and phase, while the results by the linear modal theory do not agree with the other two groups of results. The 

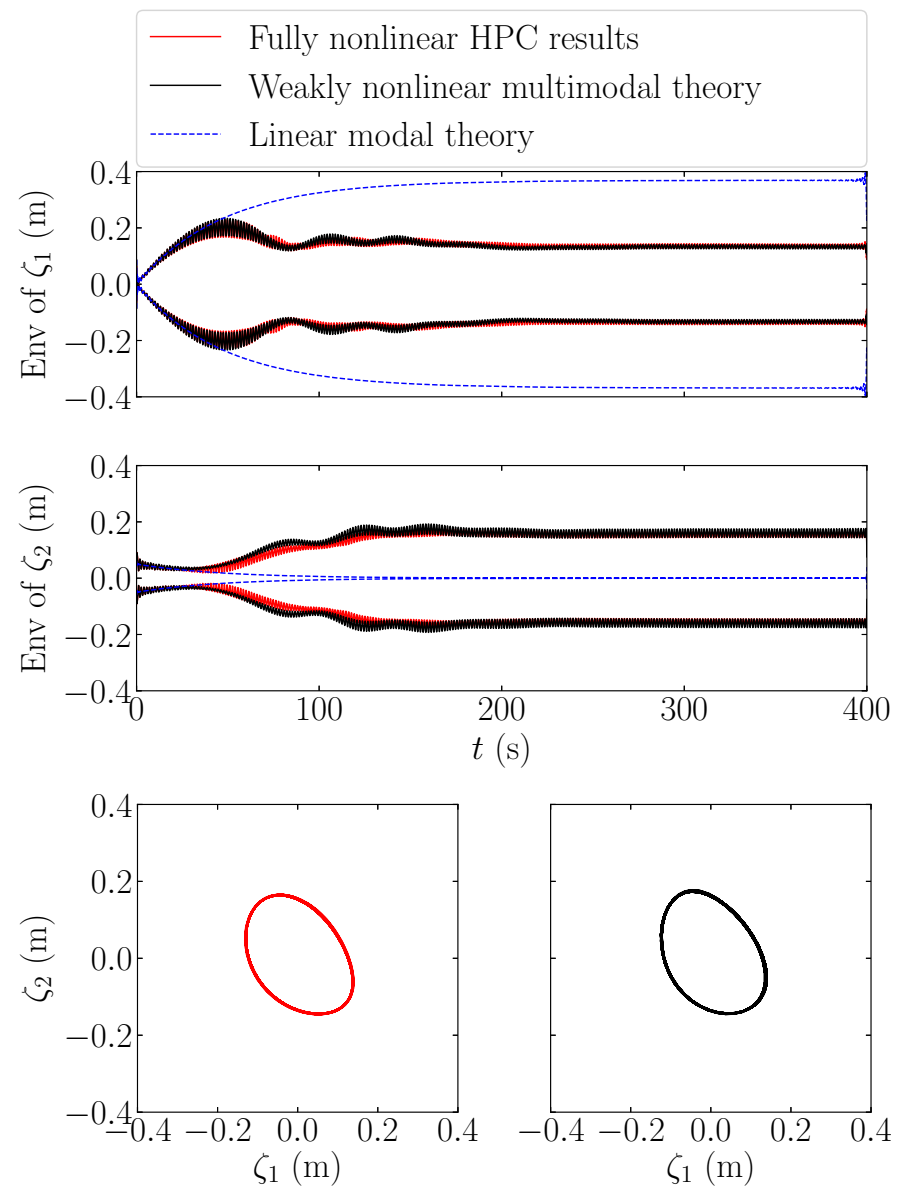

FIG. 10. Same as Fig. 8 but for $\omega=\omega_{1,1}$.

linear modal theory predicts a larger amplitude of $\zeta_{1}$ and diminishing $\zeta_{2}$. More in-depth analysis of time-harmonic swirling waves is presented in Sec. IV D.

Figures 14 and 15 present the time history of the normalized hydrodynamic forces and moments due to sloshing for all ranges of excitation frequencies considered. Again, despite the observed nonlinearity in $\zeta$, the forces and moments are remarkably linear. When swirling waves occur as shown in the second and third rows, there are lateral force and roll moment components acting on the tank in the steady state. This is particularly important for the mooring line integrity assessment in marine and aquaculture engineering.

\section{Periodically modulated swirling waves}

Periodically modulated swirling waves which occur at $\omega=0.99 \omega_{1,1}$ are further investigated in this section. The differences in both $\zeta_{1}$ and $\zeta_{2}$, as previously shown in Fig. 9, are further investigated by looking at the harmonic structures. Figures 16 and 17 show the comparison of the harmonic structures from the HPC results (top) and the weakly nonlinear multimodal theory (bottom), where the total signal is plotted in gray, the first harmonic (linear component) in red, and the difference and sum second harmonic in black for $\zeta_{1}$ and $\zeta_{2}$, respectively. It can be observed that the first harmonic components of both $\zeta_{1}$ and $\zeta_{2}$ are very similar apart from the difference in modulating (or beating) 

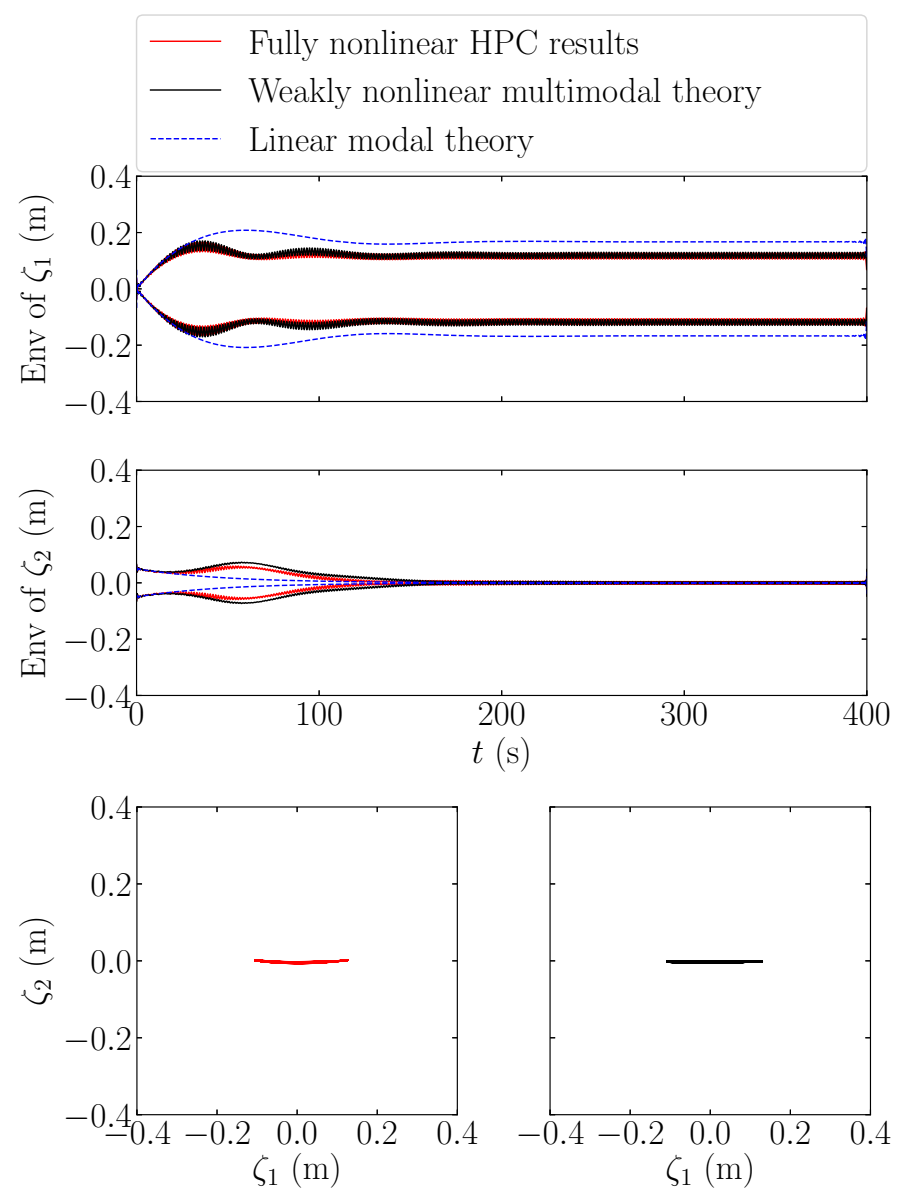

FIG. 11. Same as Fig. 8 but for $\omega=1.01 \omega_{1,1}$.

frequency, in that the weakly nonlinear multimodal theory predicts smaller beating frequency (or longer period) than the HPC results. The second harmonic component of $\zeta_{1}$ is also very similar. A more noticeable difference is observed in the second harmonic component of $\zeta_{2}$.

To investigate how the amplitude and phase change over time, we perform the short time Fourier transform (STFT) to time histories of $\zeta_{1}$ and $\zeta_{2}$ obtained from the fully nonlinear HPC method. From the variation of amplitudes in time, we observe that both the first and second harmonic components are appreciable, consistent with Figs. 16 and 17. From the variation of the phases in time, the individual phases of the first harmonic, denoted by $\varphi_{1}^{(\omega)}$ and $\varphi_{2}^{(\omega)}$ (top subplot), together with the phase difference $\varphi_{2}^{(\omega)}-\varphi_{1}^{(\omega)}$ (bottom subplot), are plotted in Fig. 18. Besides the modulating amplitudes of $\zeta_{1}$ and $\zeta_{2}$, the individual phases are also modulating, and the phase difference $\varphi_{2}^{(\omega)}-\varphi_{1}^{(\omega)}$ is slowly varying between $-\pi / 2$ and 0 . The phase difference of 0 and $-\pi / 2$ corresponds to planar waves and swirling waves, respectively, and indicates $\zeta_{2}$ as a phase lag. Therefore the modulating phase difference between $-\pi / 2$ and 0 indicates that the periodically modulated swirling waves contain both planar and swirling waves, and the wave pattern switches back and forth between oblique planar waves and anticlockwise swirling waves [37].

Comparing between Figs. 16 and 17, the second harmonic component of $\zeta_{1}$ takes up nearly $10 \%$, whereas that of $\zeta_{2}$ up to $20 \%$, indicating that the second harmonic components are non-negligible. 

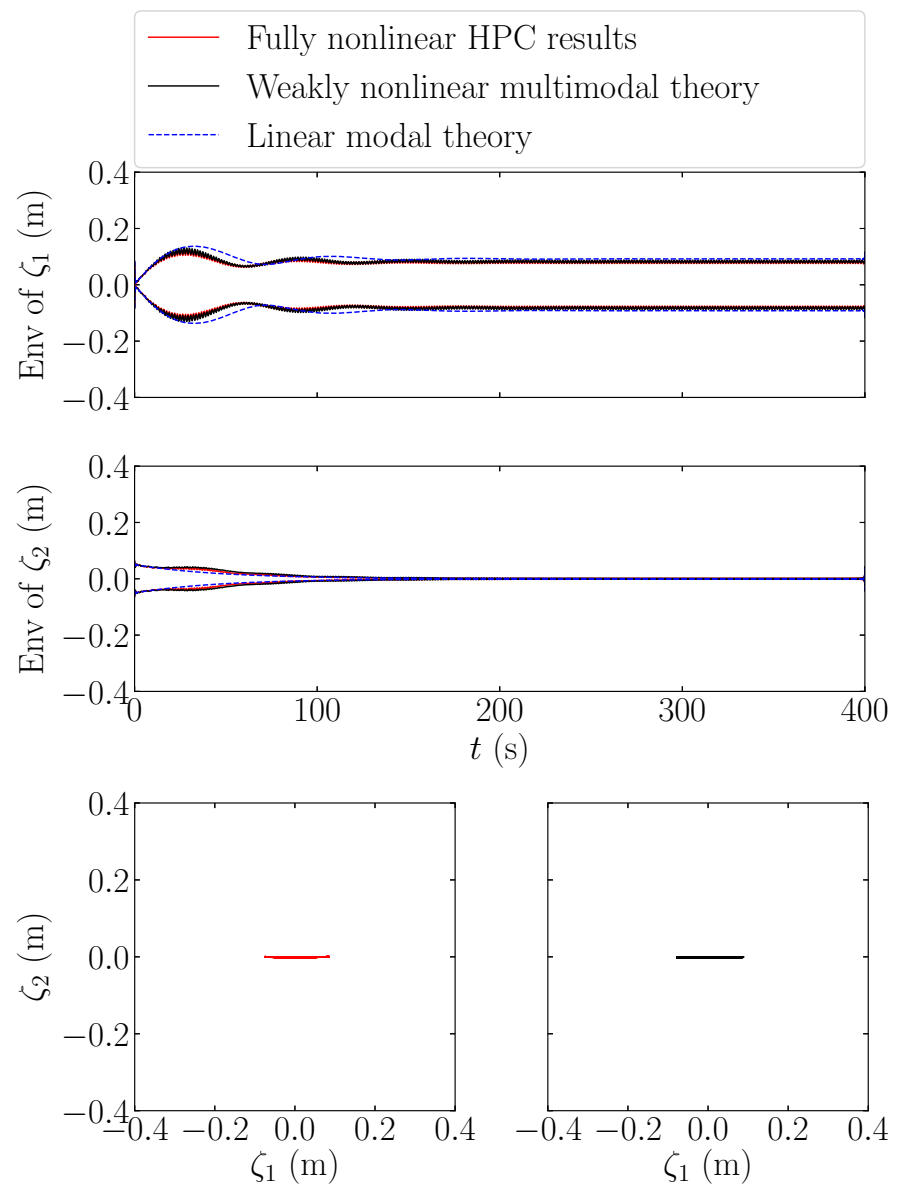

FIG. 12. Same as Fig. 8 but for $\omega=1.02 \omega_{1,1}$.

Similar to the first harmonic component, the amplitudes of the second harmonic component are also modulating with time. To reveal the behavior of the phase in time, the same STFT is performed on the second harmonic component. The time histories of individual phase and phase differences of the second harmonic components $\varphi_{1}^{(2 \omega)}$ and $\varphi_{2}^{(2 \omega)}$ are depicted in Fig. 19. It can be observed that the phases of $\zeta_{1}$ and $\zeta_{2}$ are almost in unison, and the phase difference is almost zero, except at several time instants where the amplitude of either $\zeta_{1}$ or $\zeta_{2}$ is approaching zero. Therefore the second harmonic component of the wave pattern exhibits oblique planar behavior.

To examine the influence of initial conditions, we change the phase of the initial disturbance, which is written as

$$
\zeta(r, \theta, t=0)=0.05 \frac{J_{1}\left(k_{1,1} r\right)}{J_{1}\left(k_{1,1} R\right)} \sin (\theta+\pi) .
$$

In the same manner, we perform the STFT to the time histories of $\zeta_{1}$ and $\zeta_{2}$. The time series of individual phases of the first harmonic components $\varphi_{1}^{(\omega)}$ and $\varphi_{2}^{(\omega)}$ are depicted in the top subplot of Fig. 20, and the phase difference $\varphi_{2}^{(\omega)}-\varphi_{1}^{(\omega)}$ is shown in the bottom subplot. The amplitudes of $\zeta_{1}^{(\omega)}$ and $\zeta_{2}^{(\omega)}$ for different initial conditions are comparable to those in Figs. 9 and 18 at the steady state, which demonstrate that the periodically modulated swirling behavior is robust. However, for initial 


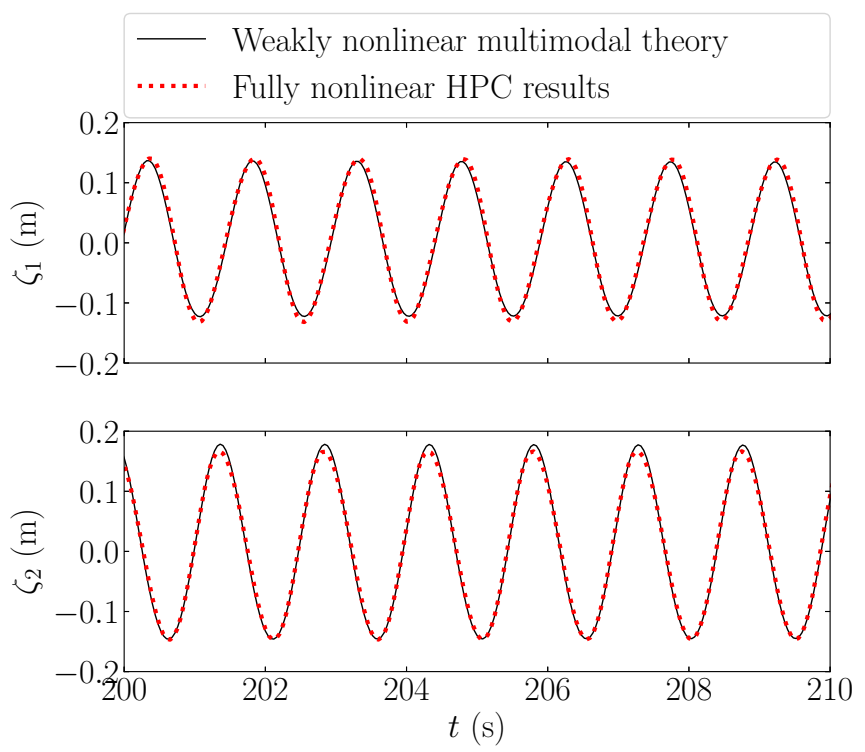

FIG. 13. Time histories of $\zeta_{1}$ and $\zeta_{2}$ for $\omega=\omega_{1,1}$ in the steady state. The present fully nonlinear HPC results (red) are in comparison with the results determined by the weakly nonlinear multimodal theory (black).

condition (24), $\zeta_{2}$ is phase lead indicating the wave pattern exhibiting clockwise swirling behavior instead [37]. Therefore, initial conditions only affect the swirling direction. It has been examined by changing the phase of the surging motion that the resultant sloshing response at the steady state is consistent.

\section{Time-harmonic swirling waves}

As a sequel to Sec. IV C, further investigation into the occurrence of time-harmonic swirling waves which occurs at $\omega=\omega_{1,1}$ is set forth in this section. Similarly, we perform the STFT to the time series of $\zeta_{1}$ and $\zeta_{2}$ at $\omega=\omega_{1,1}$, and observe that the first harmonic component is predominant and higher harmonic components are negligible. Using STFT, the temporal behavior of the amplitude and phase of the first harmonic component can be extracted. In the top subplot of Fig. 21, the time series of individual phases of the first harmonic components of $\zeta_{1}$ and $\zeta_{2}$ denoted by $\varphi_{1}^{(\omega)}$ and $\varphi_{2}^{(\omega)}$ are depicted, and the phase difference between $\zeta_{1}^{(\omega)}$ and $\zeta_{2}^{(\omega)}$, defined as $\varphi_{2}^{(\omega)}-\varphi_{1}^{(\omega)}$, is shown in the bottom subplot. In the initial stage $(t<30 \mathrm{~s})$, the phase difference is $\pi$ because of the initial condition (21). In this stage, $\zeta_{1}$ is excited by the forced surging motion, whereas $\zeta_{2}$ is undergoing free oscillation with slight decay in the amplitude. During the transition stage approximately between 30 and $100 \mathrm{~s}, \zeta_{2}$ is being built up, resulting in an increasing amplitude, which indicates that the transverse symmetric mode is being excited. The phases of $\zeta_{1}$ and $\zeta_{2}$ are also varying in time, causing the phase difference to gradually shift from $\pi$ to slightly larger than $\pi / 2$. In the steady state $t>200 \mathrm{~s}$, the amplitudes of $\zeta_{1}^{(\omega)}$ and $\zeta_{2}^{(\omega)}$ are constant, and the wave pattern exhibits clockwise swirling behavior because $\zeta_{2}$ is phase lead [37].

In the same manner we investigate the influence of initial conditions by changing the phase of the initial disturbance according to condition (24). We perform the STFT to the time histories of $\zeta_{1}$ and $\zeta_{2}$, and the individual phases of the first harmonic components and the phase difference are depicted in Fig. 22. The amplitudes and phase plots of $\zeta_{1}^{(\omega)}$ and $\zeta_{2}^{(\omega)}$ for different initial conditions are consistent with those in Fig. 21 at the steady state. This shows that initial conditions do not affect the swirling behavior or wave pattern. However, for initial condition $(24), \zeta_{1}^{(\omega)}$ 


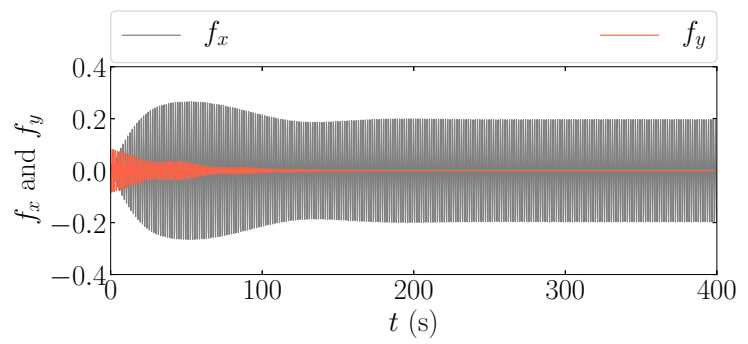

(a)

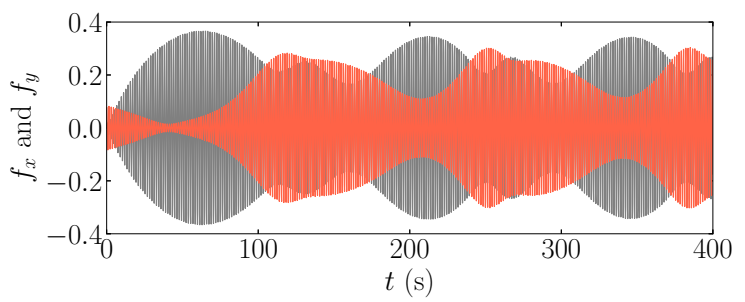

(b)

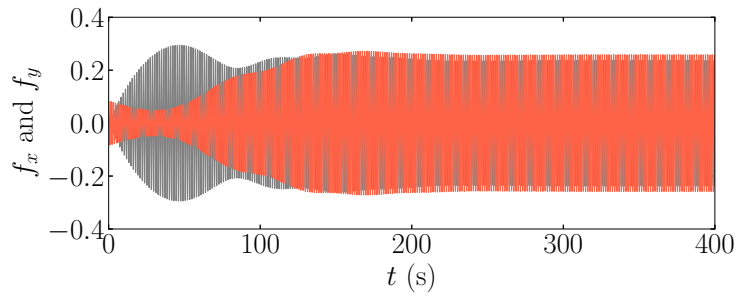

(c)

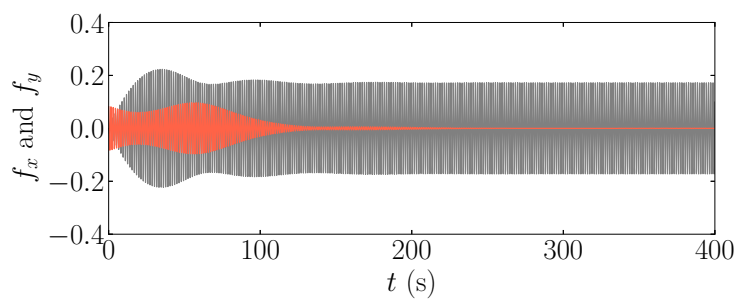

(d)

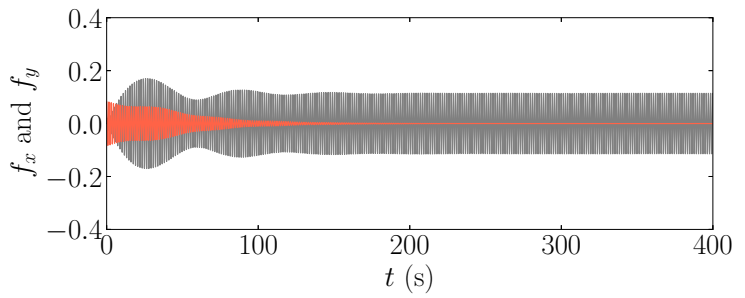

(e)

FIG. 14. Time history of the normalized hydrodynamic forces due to sloshing $\left(f_{x}=\right.$ in-line force by gray line; $f_{y}=$ lateral force by red line. (a) $\omega=0.98 \omega_{1,1}$, (b) $\omega=0.99 \omega_{1,1}$, (c) $\omega=1.00 \omega_{1,1}$, (d) $\omega=1.01 \omega_{1,1}$, and $(\mathrm{e}) \omega=1.02 \omega_{1,1}$. 


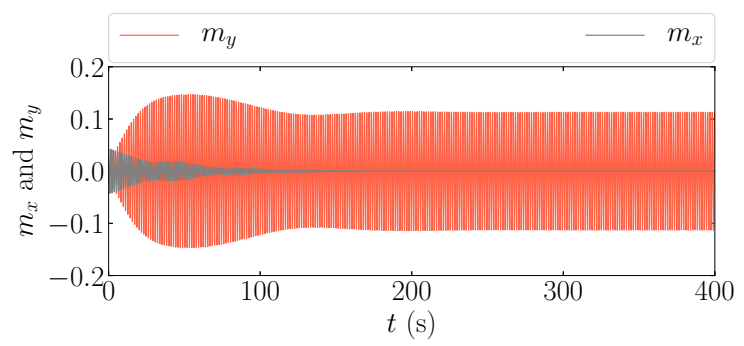

(a)

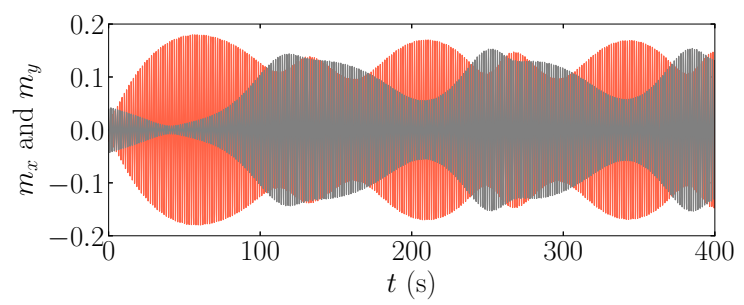

(b)

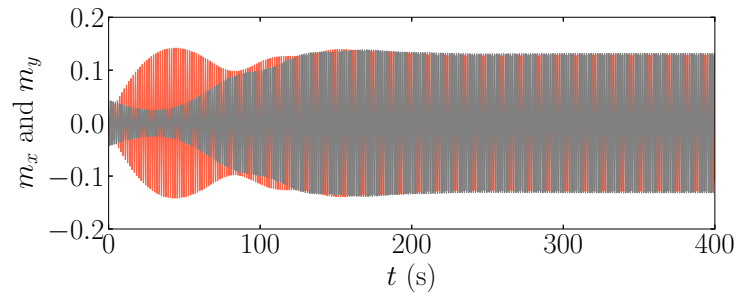

(c)

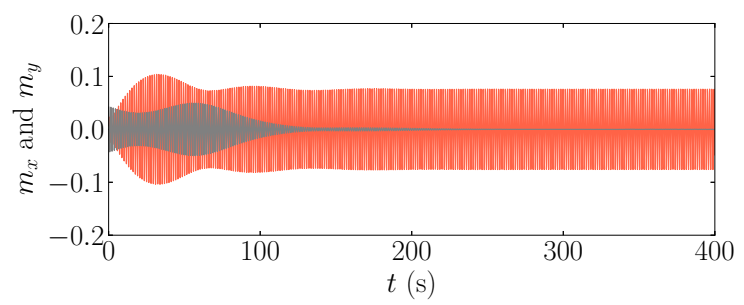

(d)

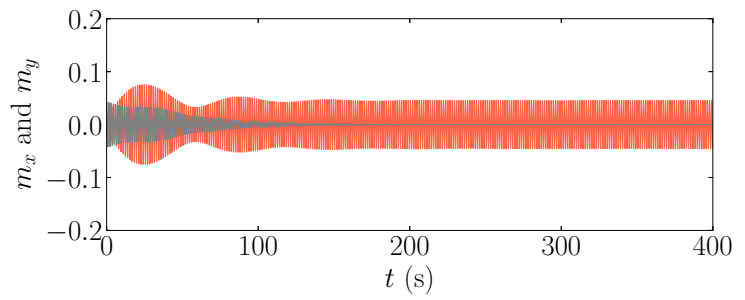

(e)

FIG. 15. Time history of the normalized hydrodynamic moments due to sloshing ( $m_{x}=$ roll moment by gray line; $m_{y}=$ pitch moment by red line). (a) $\omega=0.98 \omega_{1,1}$, (b) $\omega=0.99 \omega_{1,1}$, (c) $\omega=1.00 \omega_{1,1}$, (d) $\omega=$ $1.01 \omega_{1,1}$, and (e) $\omega=1.02 \omega_{1,1}$. 

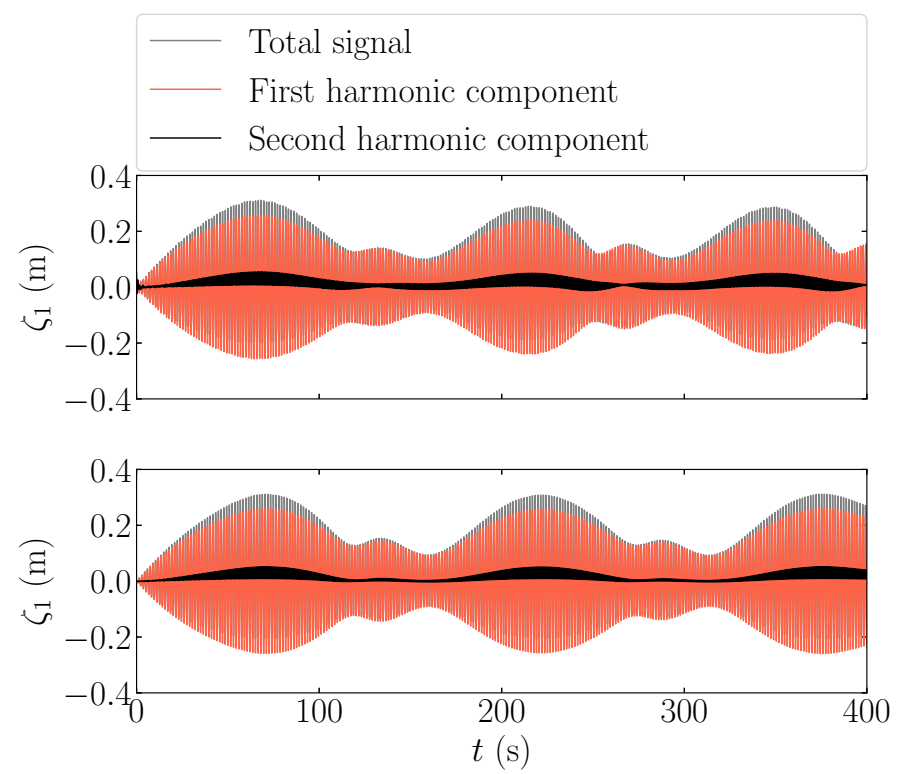

FIG. 16. Comparison of $\zeta_{1}$ at $\omega=0.99 \omega_{1,1}$ between HPC (top) and multimodal theory (bottom) in terms of the harmonic structure: total signal (gray), first harmonic (red), and second harmonic (difference and sum terms, black).

and $\zeta_{2}^{(\omega)}$ are in phase in the initial stage but gradually shift to a phase difference slightly lower than $\pi / 2$ in the steady state. Unlike Fig. $21, \zeta_{2}$ is the phase lag, in this case indicating the wave pattern exhibiting anticlockwise swirling behavior [37]. Therefore, we conclude that the
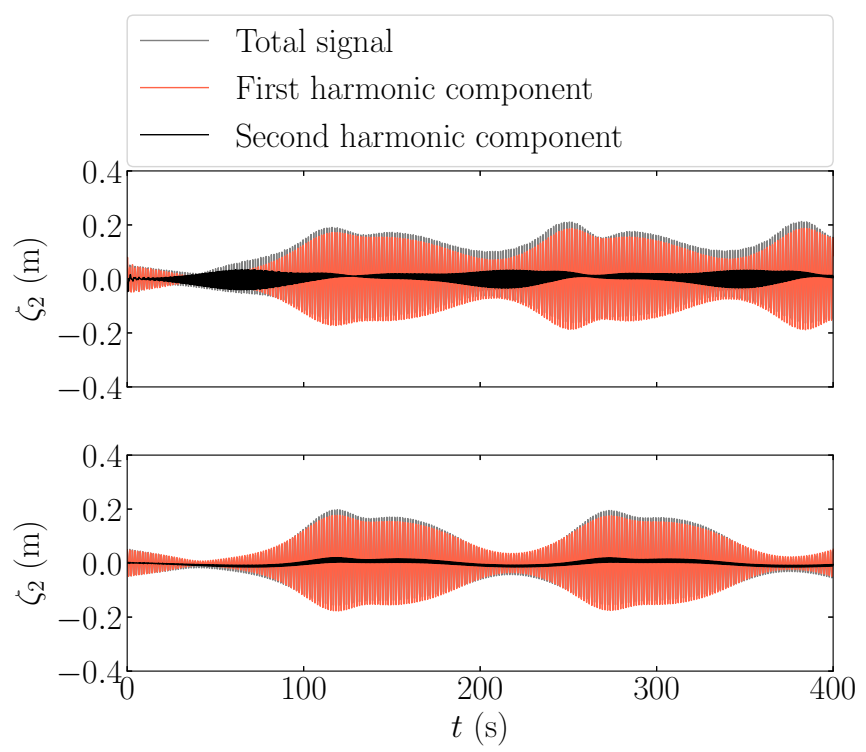

FIG. 17. Comparison of $\zeta_{2}$ at $\omega=0.99 \omega_{1,1}$ between HPC (top) and multimodal theory (bottom) in terms of the harmonic structure: total signal (gray), first harmonic (red), and second harmonic (difference and sum terms, black). 

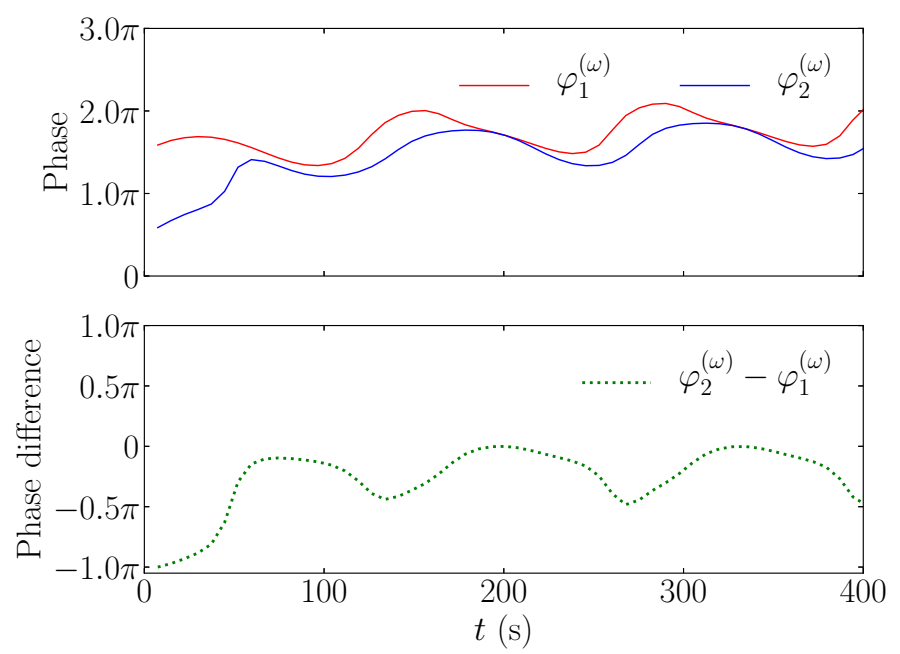

FIG. 18. Individual phases of the first harmonic components of $\zeta_{1}$ and $\zeta_{2}$ (top) as well as the phase difference (bottom) as a function of time at $\omega=0.99 \omega_{1,1} . \zeta_{1}^{(\omega)}=$ first harmonic component of $\zeta_{1} ; \zeta_{2}^{(\omega)}=$ first harmonic component of $\zeta_{2} ; \varphi_{1}^{(\omega)}=$ phase angle of $\zeta_{1}^{(\omega)} ; \varphi_{2}^{(\omega)}=$ phase angle of $\zeta_{2}^{(\omega)}$.

swirling behavior occurring in proximity to the lowest natural frequency is not dependent on initial conditions. However, initial conditions will determine the swirling direction-clockwise or anticlockwise.

We also noted that the energy exchange between the longitudinal and transverse modes leading to the occurrence of swirling waves is somewhat characterized by changes in the phase of $\zeta_{1}$. For other cases, such as $\omega=1.01 \omega_{1,1}$, the phase of $\zeta_{1}$ remains constant after the initial transient, while the amplitude of $\zeta_{2}$ is decreasing to zero (and therefore the phase of $\zeta_{2}$ is not meaningful), as shown in Fig. 23. For this case, no swirling waves are observed.
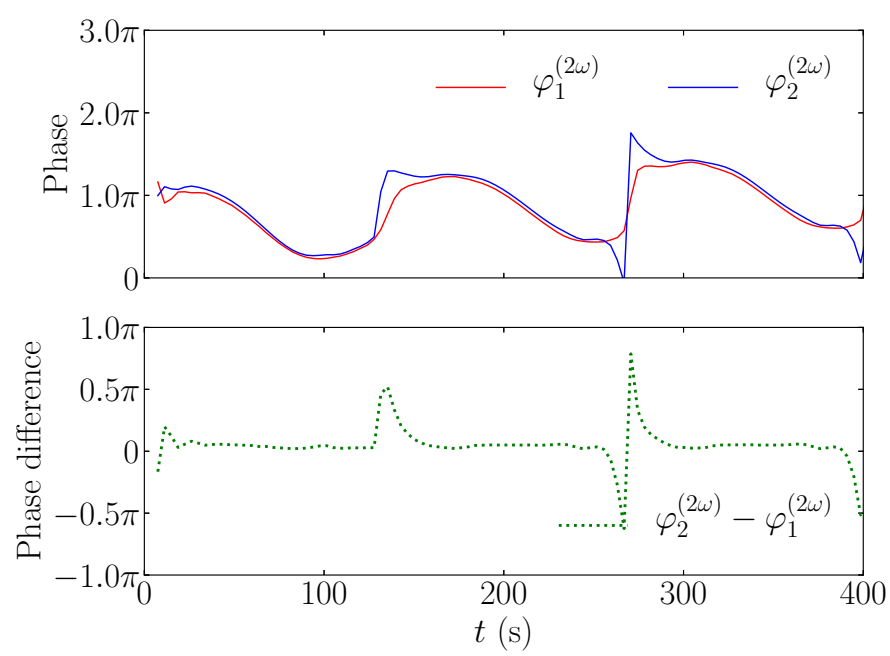

FIG. 19. Same as Fig. 18 but for the second harmonic components. $\varphi_{1}^{(2 \omega)}=$ phase angle of $\zeta_{1}^{(2 \omega)} ; \varphi_{2}^{(2 \omega)}=$ phase angle of $\zeta_{2}^{(2 \omega)}$. 

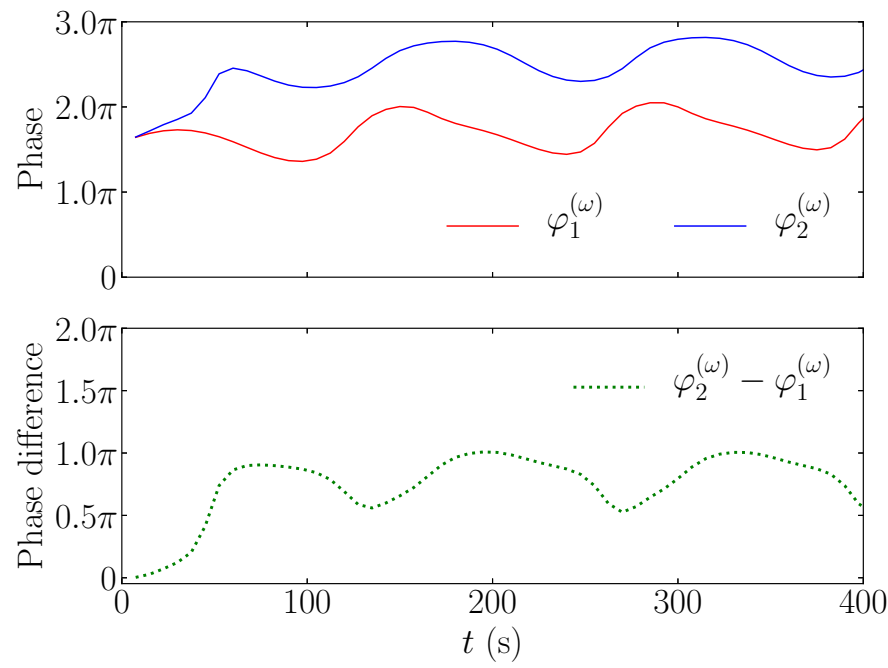

FIG. 20. Same as the bottom subplot of Fig. 18 but with the initial condition (24), respectively.

\section{TRANSIENT EXCITATION}

\section{A. NewWave-type excitation}

As a sequel to the time-harmonic oscillations, a transient compact NewWave-type excitation is considered. The average shape of the largest excitation in incident random wave field, the NewWave in surge excitation, is expressed as

$$
\eta_{N W}=\alpha_{\eta} \frac{\sum_{n} S_{\eta}\left(\omega_{n}\right) \Delta \omega \operatorname{Re}\left[\exp \left(i \omega_{n} t\right)\right]}{\sum_{n} S_{\eta}\left(\omega_{n}\right) \Delta \omega}
$$
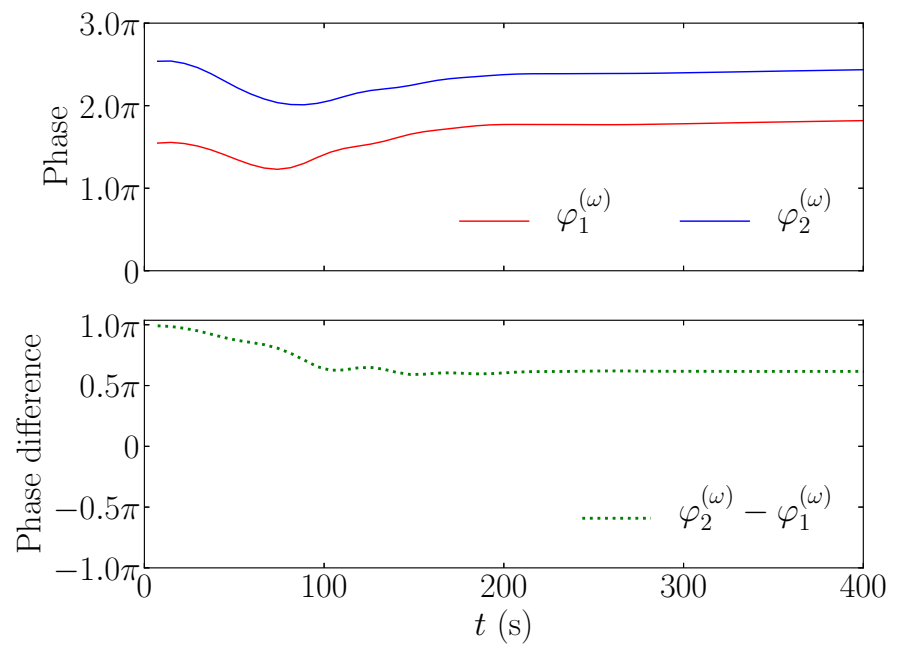

FIG. 21. Individual phases of the first harmonic components of $\zeta_{1}$ and $\zeta_{2}$ (top) as well as the phase difference (bottom) as a function of time at $\omega=\omega_{1,1} \cdot \zeta_{1}^{(\omega)}=$ first harmonic component of $\zeta_{1} ; \zeta_{2}^{(\omega)}=$ first harmonic component of $\zeta_{2} ; \varphi_{1}^{(\omega)}=$ phase angle of $\zeta_{1}^{(\omega)} ; \varphi_{2}^{(\omega)}=$ phase angle of $\zeta_{2}^{(\omega)}$. 

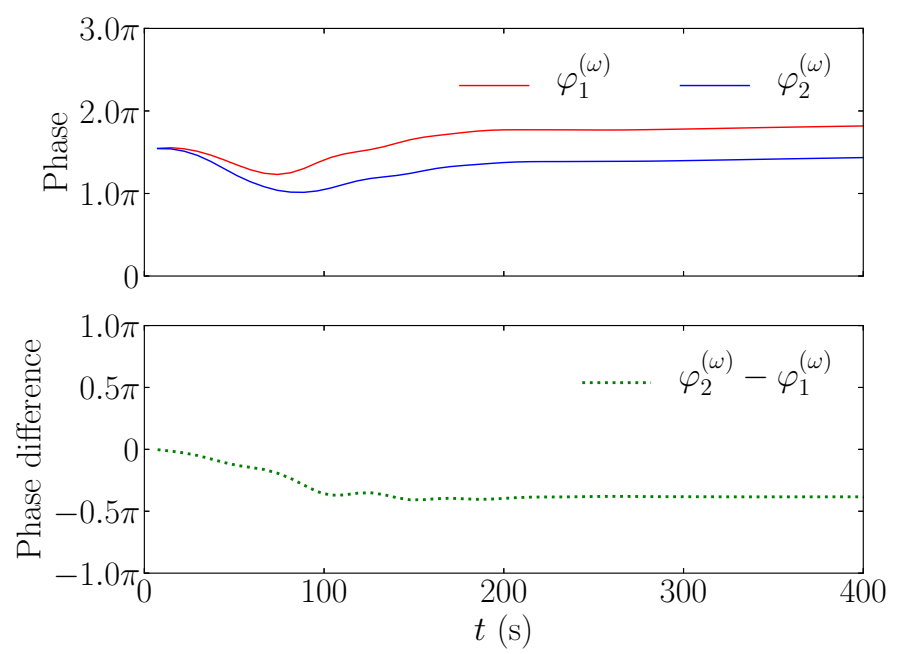

FIG. 22. Same as the bottom subplot of Fig. 21 but with the initial condition (24).

where $\alpha_{\eta}$ is the most probable extreme excitation in $N$ samples. For large $N$, the following approximation holds for a Gaussian process:

$$
\alpha_{\eta}=\sqrt{2 m_{0} \log N}
$$

where $m_{0}$ is the total variance of the spectrum, $S_{\eta}\left(\omega_{n}\right)$, which is given by $m_{0}=\sum_{n=0}^{\infty} S_{\eta}\left(\omega_{n}\right) \Delta \omega$, where $\Delta \omega$ is the differential element of the wave frequency. This result is well known [38, p. 319].

Note the use of the power spectrum, $S\left(\omega_{n}\right)$, in the above equation, which means that the amplitude spectrum of the NewWave has the same shape as the power spectrum of the underlying sea state. The NewWave temporal shape is different from that of a transient focused wave, generally used to represent extreme (or freak) waves, which is based on the amplitude spectrum of the underlying sea state (and hence the resulting temporal shape is more broadband).

Figure 24 presents the NewWave type of excitation $\eta_{N W}(t)$ and the resulting responses at two probe locations $\left(\zeta_{1}\right.$ and $\left.\zeta_{2}\right)$ in terms of surface elevation, and normalized horizontal hydrodynamic forces $\left(f_{x}, f_{y}\right)$ and moments $\left(m_{x}, m_{y}\right)$ acting on the tank, based on the assumed JONSWAP (Joint North Sea Wave Project) spectral shape with $H_{s}=0.0087 \mathrm{~m}, T_{p}=2 \pi / \omega_{1,1}=1.48 \mathrm{~s}$, and $\gamma=3.3$. Note the NewWave is made to focus at $t=10 \mathrm{~s}$. In terms of $\zeta_{1}$ in particular, it can be observed that for $t \leqslant 20 \mathrm{~s}$ the excitation is driving energy into the liquid in the tank, and after the excitation ends, the liquid undergoes free oscillation, with a decay rate commensurate with the imposed artificial damping. Similar behavior is observed for the hydrodynamic force and moment on the tank. Both

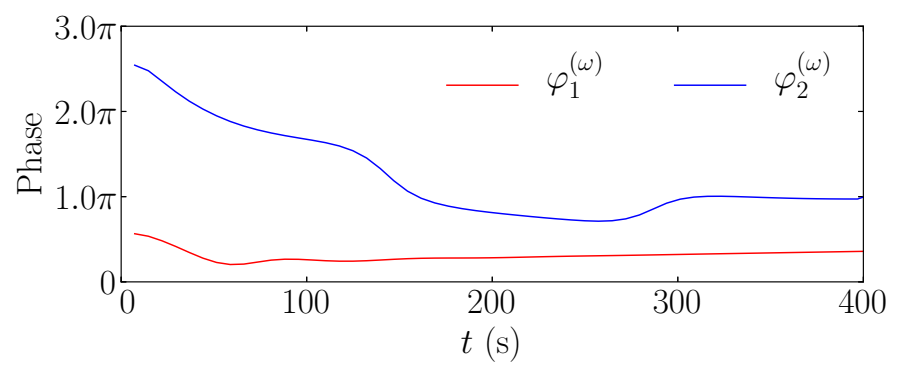

FIG. 23. Same as the bottom subplot of Fig. 21 but for $\omega=1.01 \omega_{1,1}$. 


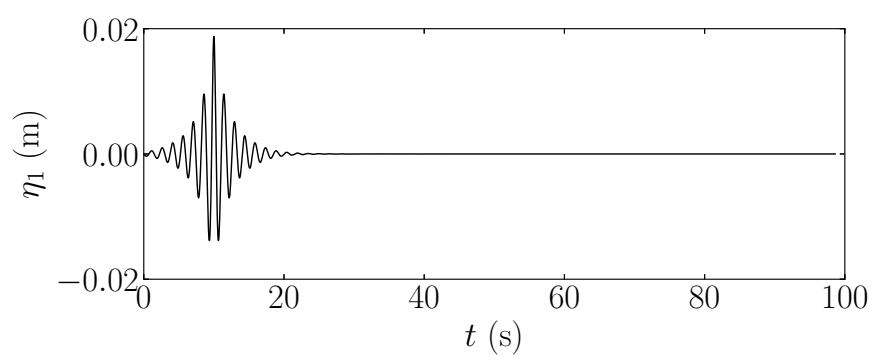

(a)

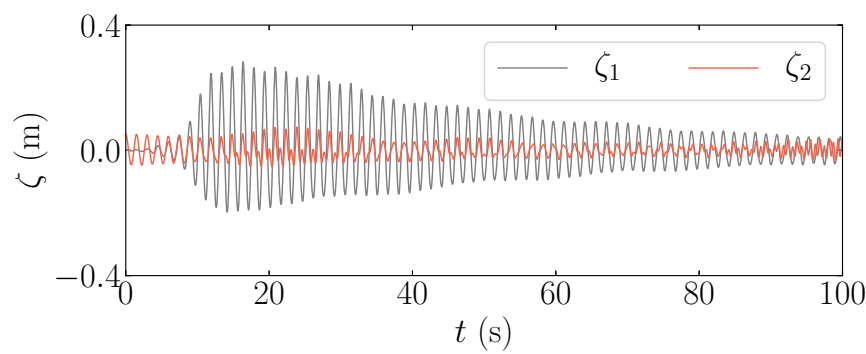

(b)

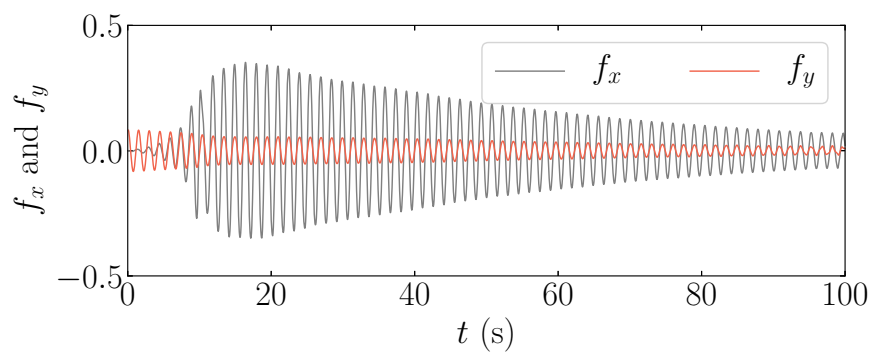

(c)

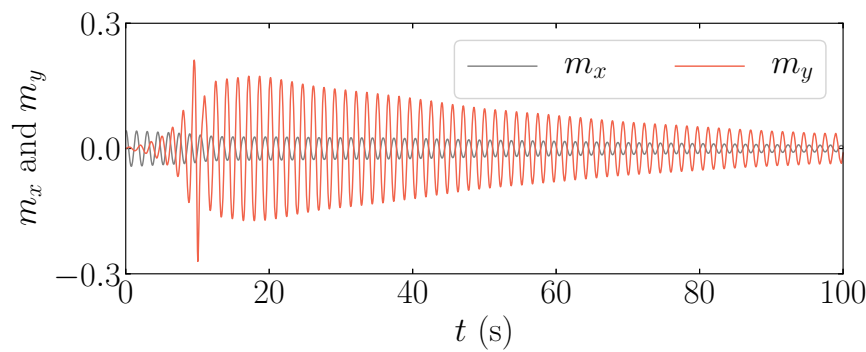

(d)

FIG. 24. Time history of a NewWave type of excitation (a) and the resulting responses in terms of surface elevation (b), hydrodynamic forces (c) and moments (d) on the tank.

responses are considered narrow band. Moreover, the amplitude of $\zeta_{2}$ is slightly increasing while that of $\zeta_{1}$ is decreasing, indicating that there is energy transfer. However, it is worth noting that $\zeta_{2}$ is induced by the initial disturbance, and it is observed that swirling waves are not induced because $\zeta_{1}$ and $\zeta_{2}$ are in phase as shown in the second subplot. Therefore, the energy transfer is not strong enough to excite swirling waves in this problem. Besides, it indicates that the appearance of the swirling waves requires a long time and continuous excitation. Comparing the harmonic structures 


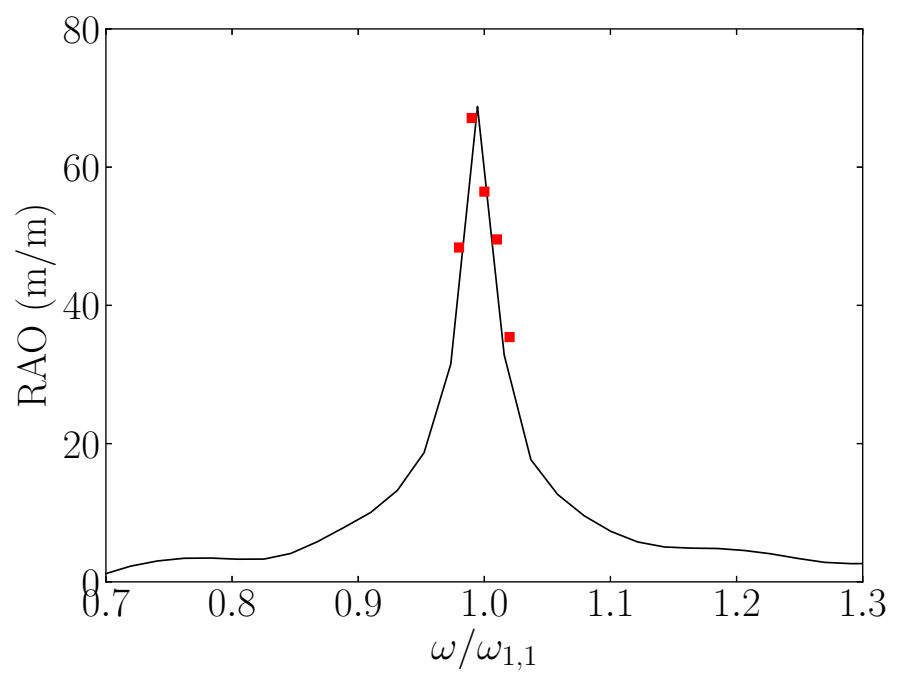

FIG. 25. Comparison of RAO derived from the NewWave type of excitation (solid line) and the periodic type of excitation (squares).

for $\zeta$ and $f$ as well as $m$, it can be seen that $\zeta$ contains higher wave harmonic components in particular for $\zeta_{1}$, and there is occurrence of secondary peaks for $\zeta_{2}$, while $f$ and $m$ are predominantly linear without any obvious vertical asymmetry. Besides, $f_{x}$ and $f_{y}$ are in phase, whereas $m_{x}$ and $m_{y}$ have the phase difference of $\pi$. At the focal time instant, an impulse in the pitch moment is observed, and the amplitude is slightly higher than the subsequent amplitudes during the free oscillation.

For problems such as liquid sloshing in a tank, the largest response may not necessarily be due to the largest wave crest or excitation. Here we present a methodology to obtain the most probable maximum response of the liquid sloshing in a realistic sea-state condition, assuming linear motion. The method was previously applied to the M4 wave energy converter to derive the most probable extreme hinge angle response for survivability assessment [39]. First, a transfer function akin to a response amplitude operator (RAO), which describes the linear relationship on the sloshing response for a given input excitation $\eta$, can be defined as

$$
|Z|=\sqrt{\frac{S_{\text {response }}}{S_{\eta}}} .
$$

For a sloshing response in $\zeta$, for example, its power spectrum can be expressed as

$$
S_{\zeta}\left(\omega_{n}\right)=S_{\eta}\left(\omega_{n}\right)\left|Z\left(\omega_{n}\right)\right|^{2} .
$$

Figure 25 shows the computed RAO from the NewWave type of excitation (solid line). Also shown are the individual RAOs derived from the periodic type of excitation for forcing frequencies ranging between $\omega=0.98 \omega_{1,1}$ and $\omega=1.02 \omega_{1,1}$ (squares), where good agreement can be seen. The RAO curve is linear within the most energetic frequency excitation range (from $\omega / \omega_{1,1}=0.7-1.3$ ), with some nonlinearity contaminating the curve to the left and right side of the peak.

From Eq. (25), the response in $\zeta$ for a given NewWave-type excitation in surge, now termed $\zeta_{\eta_{N W}}$, can be obtained from

$$
\zeta_{\eta_{N W}}=\alpha_{\eta} \frac{\sum_{n} S_{\eta}\left(\omega_{n}\right) \Delta \omega \operatorname{Re}\left[Z\left(\omega_{n}\right) \exp \left(i \omega_{n} t\right)\right]}{\sum_{n} S_{\eta}\left(\omega_{n}\right) \Delta \omega} .
$$


The largest response, termed NewWave in response, is therefore simply

$$
\zeta_{N W}=\alpha_{\zeta} \frac{\sum_{n} S_{\zeta}\left(\omega_{n}\right) \Delta \omega \operatorname{Re}\left[\exp \left(i \omega_{n} t\right)\right]}{\sum_{n} S_{\zeta}\left(\omega_{n}\right) \Delta \omega}
$$

where $\alpha_{\zeta}$ is the 1 in $N$ linear crest elevation of sloshing in the random sea state. The designer wave which would give rise to the NewWave in response is given as

$$
\eta_{\zeta_{N W}}=\alpha_{\zeta} \frac{\sum_{n} S_{\zeta}\left(\omega_{n}\right) \Delta \omega \operatorname{Re}\left[Z\left(\omega_{n}\right)^{-1} \exp \left(i \omega_{n} t\right)\right]}{\sum_{n} S_{\zeta}\left(\omega_{n}\right) \Delta \omega} .
$$

The designer wave for in-line force $f_{x}$ and pitch moment $m_{y}$ on the tank follow the same procedure.

Figure 26 presents the designer-wave type of excitation $\left(\eta_{\zeta_{N W}}\right)$ and the resulting extreme response $\left(\zeta_{N W}\right)$, as well as the associated force and moment $(f$ and $m)$. Note the peak extreme response is made to occur at $t=40 \mathrm{~s}$. Note also that the peak response is chosen to be comparable to the previous response due to NewWave-type excitation; however, the energy contained in the designerwave excitation is $30 \%$ of that contained in the NewWave-type excitation. If the same energy is used, the resulting extreme response will be much larger. This illustrates the potential application of a designer wave to analyze the extreme response of a floating system when survivability is in question.

It can be seen from the figure that before the peak response, the designer-wave excitation causes a buildup of $\zeta_{1}$. After the excitation ends, the sloshing liquid undergoes free vibration. Since then, $\zeta_{1}$ gradually decreases whereas $\zeta_{2}$ increases, indicating that there is energy transfer from the longitudinal symmetric mode to transverse symmetric mode. After around six periods, however, both $\zeta_{1}$ and $\zeta_{2}$ decay due to the absence of the external excitation, and $\zeta_{1}$ and $\zeta_{2}$ are in phase, indicating that the wave pattern exhibits oblique planar behavior rather than swirling waves. Similar behavior is observed for $f_{x}$ and $m_{y}$. In fact, both $\zeta\left(\zeta_{1}\right.$ and $\left.\zeta_{2}\right)$ and $f\left(f_{x}\right.$ and $\left.f_{y}\right)$ are in phase, whereas $m_{x}$ and $m_{y}$ are $\pi$ apart, similar to Fig. 24. Furthermore, $\zeta_{1}, f_{x}$, and $m_{y}$ are slowly varying in amplitude, and therefore the designer waves for the other types of responses are very similar. $\zeta_{2}$, on the other hand, is due to mode interaction and not directly associated with the induced surge motion or excitation; therefore the designer-wave excitation for $\zeta_{2}$ does not exist. Neither do $f_{y}$ and $m_{x}$.

\section{B. Focused-wave-type excitation}

In addition to the time-harmonic and NewWave-type oscillations, an impulsive focused-wavetype excitation is considered with the energetic frequency coinciding with the lowest natural frequency, representing an extreme or freak wave type of event. To generate a localized focused wave group, a set of $N$ Fourier wave components is generated at an upstream location at the beginning following an assumed wave spectrum (such as JONSWAP). At a predefined location downstream at a later time, all the wave components are intended to combine into a typically crest-focused wave group (defined as having a horizontal symmetry between the adjacent troughs on either side of the largest crest in time).

For a given power spectrum of the excitation, $S_{\eta}(\omega)$, which is discretized over $N+1$ wave components, the following transformation into the amplitude spectrum is applied:

$$
A_{\eta}\left(\omega_{j}\right)=\sqrt{\left[S_{\eta}\left(\omega_{j+1}\right)+S_{\eta}\left(\omega_{j}\right)\right] \cdot\left(\omega_{j+1}-\omega_{j}\right)} .
$$

The temporal shape of a crest-focused wave group made to focus at $t_{f}$ can be expressed as

$$
\eta_{C F}(t)=\sum_{j=1}^{N} A_{\eta}\left(\omega_{j}\right) \cdot \operatorname{Re}\left[\mathrm{e}^{i \omega_{j}\left(t-t_{f}\right)}\right] \Delta \omega .
$$

An example of a crest-focused wave group type of excitation is shown in the top row of Fig. 27, with assumed JONSWAP spectral shape with $H_{s}=0.0087 \mathrm{~m}, T_{p}=1.48 \mathrm{~s}$, and $\gamma=3.3$. The resulting responses in terms of free-surface elevation at two probed locations $\left(\zeta_{1}\right.$ and $\left.\zeta_{2}\right)$ as 


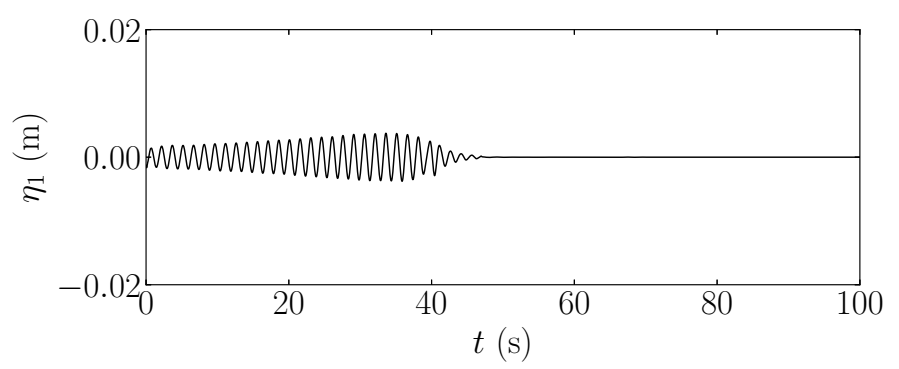

(a)

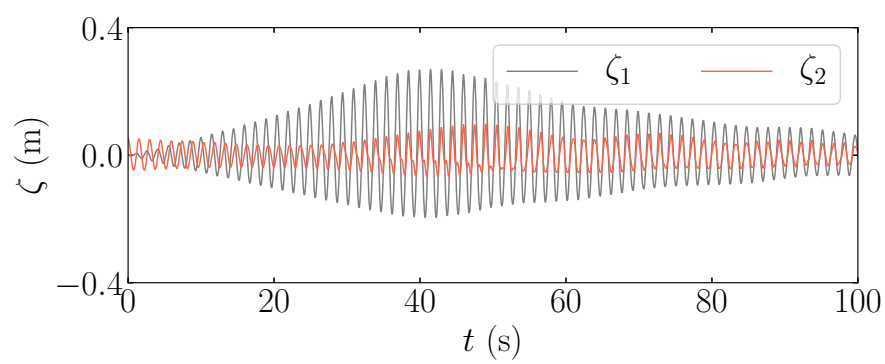

(b)

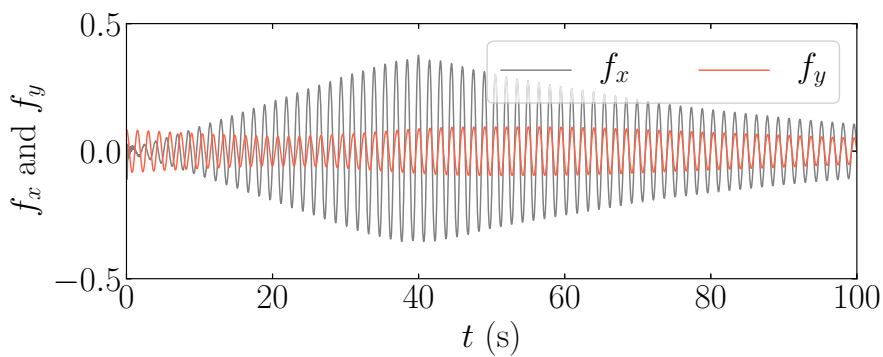

(c)

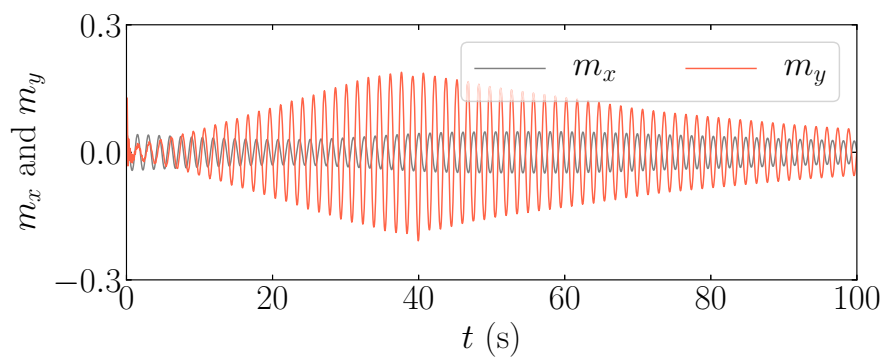

(d)

FIG. 26. Time history of a designer-wave-type excitation (a) and the resulting responses in terms of surface elevation (b), hydrodynamic force (c) and moment (d) on the tank.

well as the normalized hydrodynamic forces $\left(f_{x}, f_{y}\right)$ and moments $\left(m_{x}, m_{y}\right)$ are exhibited from the second to the bottom rows of the same figure. The in-line force and pitch moment exhibits a strong impulselike feature which was not observed in the previous subsection. Meanwhile, the liquid sloshing response is similar to that induced by NewWave-type excitation, despite the larger or more impulsive excitation in this case. 


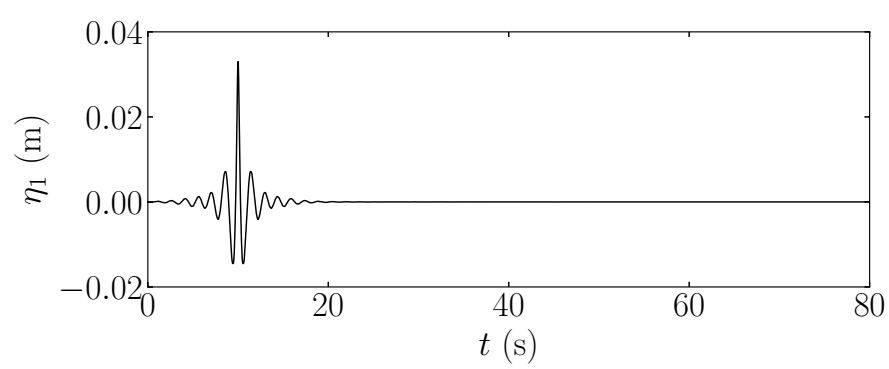

(a)

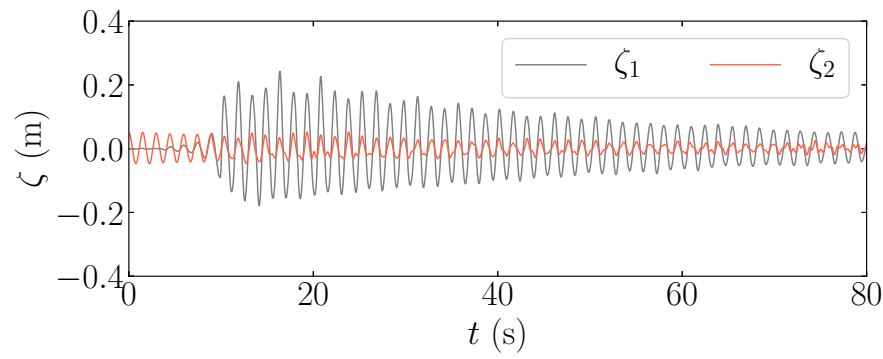

(b)

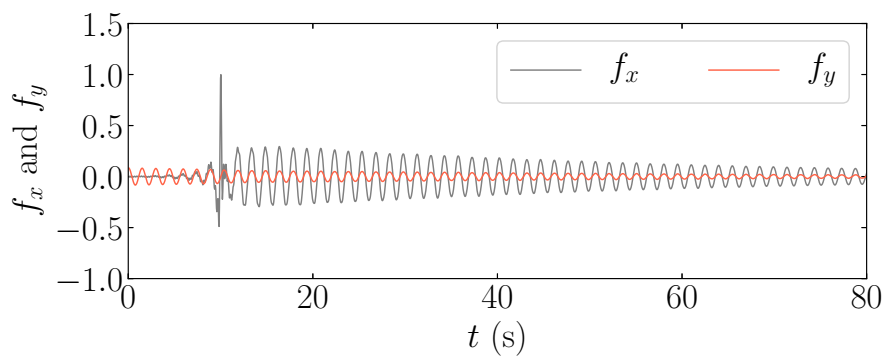

(c)

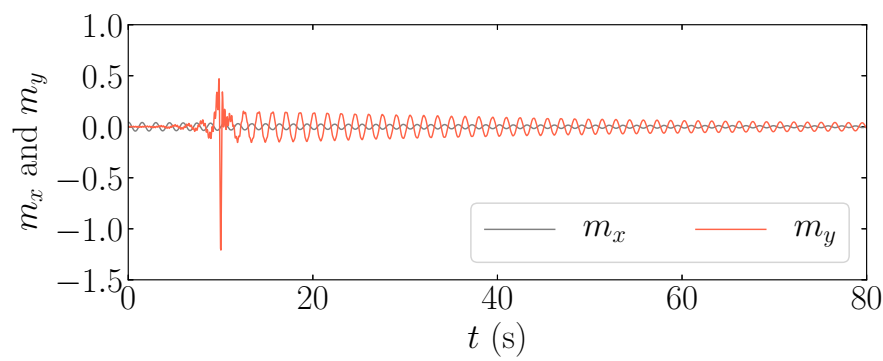

(d)

FIG. 27. Time history of a crest-focused type of excitation (a) and the resulting responses in terms of surface elevation (b), hydrodynamic force (c) and moment (d) on the tank.

To interpret the impulselike feature in the in-line force and pitch moment, we decompose the total velocity potential $\Phi$ into [6]

$$
\Phi(\vec{x}, t)=\dot{\eta}_{1} x+\phi(\vec{x}, t)
$$

where $\phi$ is the potential due to sloshing, and the first term is associated with the tank's surging motion. Then the linear term of the hydrodynamic force can be decomposed into two components 

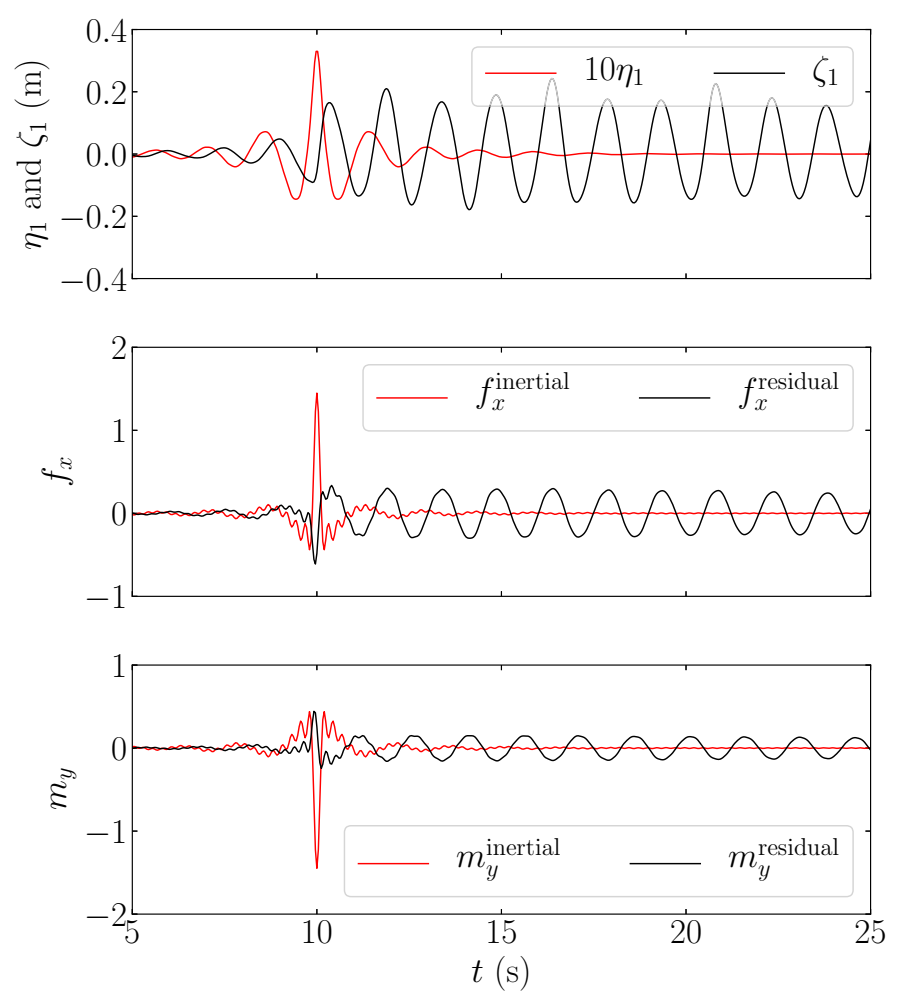

FIG. 28. Time history of excitation and sloshing response in $\zeta_{1}$ (top) and decomposed hydrodynamic force and moment into inertial and residual components (middle and bottom) for focused-wave-type excitation.

associated with the tank's motion and sloshing. Then the in-line force $F_{x}$ can be written as

$$
\begin{aligned}
F_{x} & =-\rho \iint_{S} \frac{\partial \Phi}{\partial t} n_{x} d S+[\text { nonlinear terms] } \\
& =\underbrace{-\mathcal{M} \ddot{\eta}_{1}}_{\text {inertial }} \underbrace{-\rho \iint_{S} \frac{\partial \phi}{\partial t} n_{x} d S+\text { [nonlinear terms] }}_{\text {residual }},
\end{aligned}
$$

where $\mathcal{M}$ means the mass of the liquid inside of the tank, and the pitch moment $M_{y}$ is written as

$$
\begin{aligned}
M_{y} & =-\rho \iint_{S} \frac{\partial \Phi}{\partial t}\left(z n_{x}-x n_{z}\right) d S+[\text { nonlinear terms] } \\
& =\underbrace{\frac{\mathcal{M} h}{2} \ddot{\eta}_{1}}_{\text {inertial }} \underbrace{-\rho \iint_{S} \frac{\partial \phi}{\partial t}\left(z n_{x}-x n_{z}\right) d S+[\text { nonlinear terms] }]}_{\text {residual }} .
\end{aligned}
$$

In the vicinity of the focal time instant $t=t_{f}=10 \mathrm{~s}$, the acceleration of the tank is increased abruptly. This induces a sudden increase in the inertial force and the pitch moment, which results in the peak observed in the normalized in-line force $f_{x}$ and pitch moment $m_{y}$. In order to confirm this, we separate the inertial components $f_{x}^{\text {inertial }}$ and $m_{y}^{\text {inertial }}$ from the total in-line force $f_{x}$ and pitch moment $m_{y}$ as depicted in Fig. 28. At the focal time instant $t=t_{f}=10 \mathrm{~s}$, the inertial component dominates the total force and moment. If one is interested in the wave that causes the largest force in this example, the designer wave will look very similar to the crest-focused-wave type of excitation. 

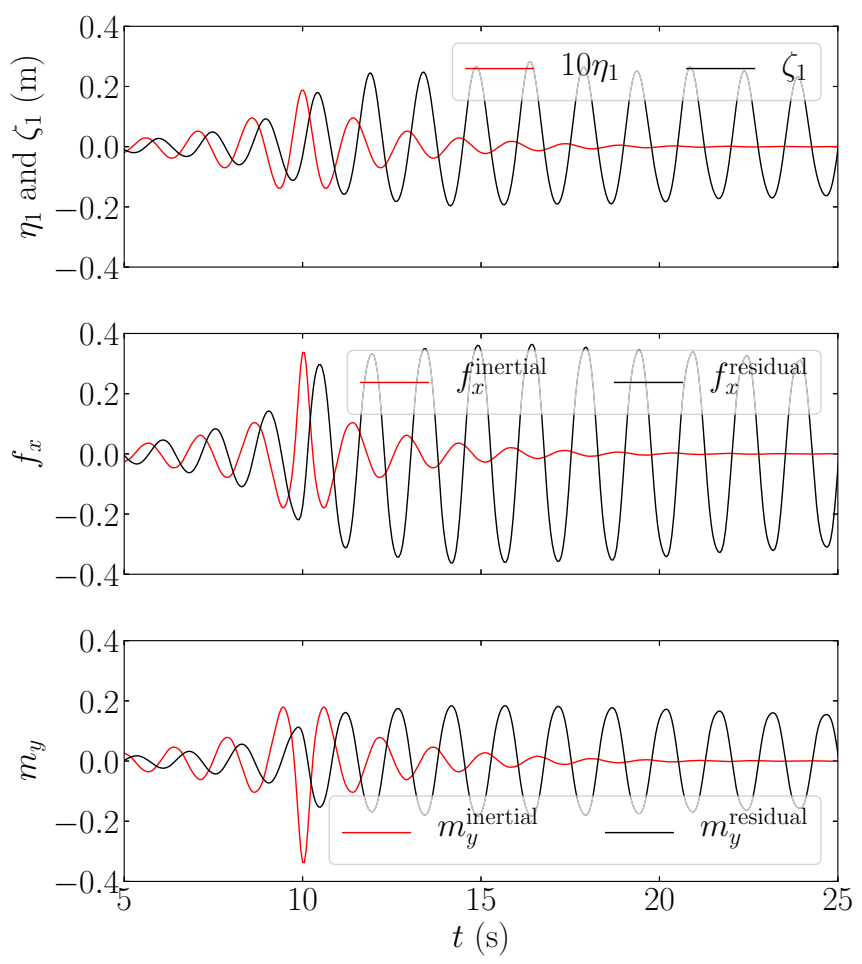

FIG. 29. Same as Fig. 28 but for NewWave-type excitation.

This is in contrast to the force and moment components due to the NewWave-type excitation, which is shown in Fig. 29. Both the inertial and residual forces are of comparable magnitude, and therefore the total hydrodynamic force and moment do not exhibit obvious impulselike feature. In the absence of liquid sloshing in the circular tank, the designer wave will follow the same shape as the NewWave-type excitation. However, due to the effect of liquid sloshing in the tank, which produces an additional residual force and moment comparable to the inertial component induced by the excitation, the NewWave-type excitation may not yield the largest force. The required excitation, which is the designer-wave-type excitation, is as shown in the top subplot in Fig. 26, and the decomposed force and moment are plotted in the middle and bottom subplots in Fig. 30. It can be observed that the designer-wave-type excitation minimizes the induced inertial component and maximizes the liquid sloshing in the tank, represented by $\zeta_{1}$, which in turn maximizes the residual component in the total force and moment.

\section{CONCLUDING REMARKS AND FUTURE PERSPECTIVES}

A fully nonlinear HPC method based on the overset mesh is developed to delve into the flow physics associated with liquid sloshing in an upright circular base tank under the surging motion. Both periodic and transient-type excitations are considered.

When the tank is under periodic excitation in proximity to the lowest resonant frequency, planar and nonplanar wave patterns, including time-harmonic and periodically modulated swirling waves, are excited after a long buildup time. For the occurrence of swirling waves, the phase difference gradually shifts to nearly $\pi / 2$. During the transition stage, the amplitude of $\zeta_{2}$ is increasing under the effect of mode interactions. The occurrence of swirling waves does not depend on initial conditions, but the initial condition determines the swirling direction: clockwise or anticlockwise. The periodically modulated swirling waves switch back and forth between planar 

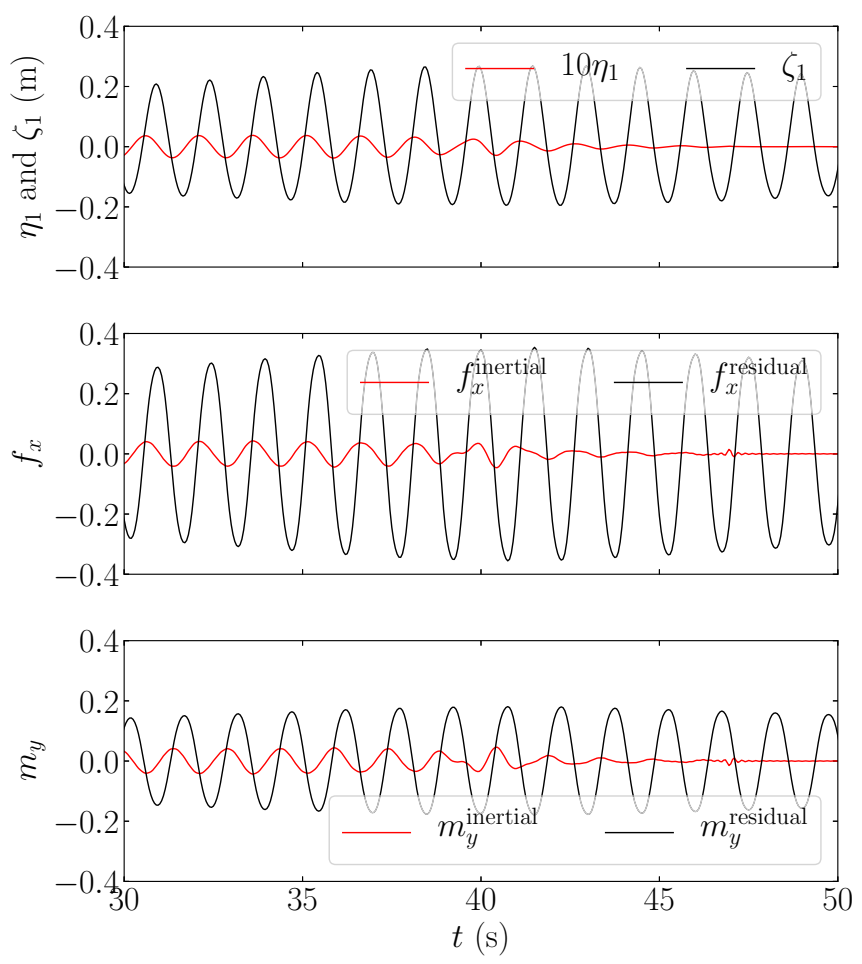

FIG. 30. Same as Fig. 28 but for designer-wave-type excitation.

waves and swirling waves. The sloshing response in terms of free-surface elevation as well as hydrodynamic force and moment are examined. The surface elevation response time series exhibits nonlinearities with a higher crest and lower trough, while the force and moment response time series are remarkably linear without appreciable vertical asymmetry. The responses are compared with analytical predictions from the linear modal theory and the weakly nonlinear multimodal theory. In general, good agreement between the fully nonlinear HPC method and the weakly nonlinear multimodal theory is observed. The disagreement with the linear modal theory indicates that the occurrence of nonplanar wave patterns is due to mode interactions. When nonplanar waves occur, the lateral force and roll moment components are experienced by the tank, which could be important for mooring line integrity assessment in marine and aquaculture engineering.

Transient responses are examined using NewWave- and focused-wave-type excitations. For both transient-type excitations, narrow-banded responses are observed. Despite the difference in the focused amplitudes, the free-surface elevations induced by both excitations are very similar, and only a planar wave pattern could be excited. However, the hydrodynamic forces can be quite different. An impulselike feature is observed for the in-line force and pitch moment induced by focused-wave-type excitation. Based on the results from NewWave-type excitation, the designerwave-type excitation is applied to the liquid sloshing problem to yield the largest sloshing responses, both in free-surface elevation as well as in-line hydrodynamic force and moment acting on the tank. Further investigation by force decomposition reveals that the inertial component is dominant under focused-wave-type excitation, while the residual component is energized to be more dominant under designer-wave-type excitation.

Having accounted for periodic and transient-type excitations in the present study, the sloshing response under the influence of random waves and wave grouping phenomena that can occur in a real ocean may be all different. In addition, since the present numerical model adopts the free- 
surface-fitted grid and a semi-Lagrangian scheme, the free-surface profile must be a single-valued function, and therefore this study is limited to small-amplitude oscillations. For a relatively largeamplitude motion, local wave breaking occurs [11], and techniques to cope with breaking waves must be incorporated. All these will be investigated in future work.

\section{ACKNOWLEDGMENT}

This research is supported by A*STAR Science and Engineering Research Council, Grant No. 17219 00089, under the Marine \& Offshore Strategic Research Programme (M\&O SRP).

[1] O. M. Faltinsen, Sea Loads on Ships and Offshore Structures (Cambridge University Press, Cambridge, England, 1993).

[2] Y. Tan, Y. Shao, and R. Read, Coupled motion and sloshing analysis of a rigid cylindrical closed fish cage in regular waves, in Proceedings of the 38th International Conference on Ocean, Offshore and Arctic Engineering, Glasgow, Scotland, UK (American Society of Mechanical Engineers Digital Collection, 2019).

[3] H. Hernández-Barrios, E. Heredia-Zavoni, and Á. A. Aldama-Rodríguez, Nonlinear sloshing response of cylindrical tanks subjected to earthquake ground motion, Eng. Struct. 29, 3364 (2007).

[4] H. N. Abramson, The dynamic behavior of liquids in moving containers, with applications to space vehicle technology, NASA Technical Repport SP-106 (1966).

[5] R. A. Ibrahim, Liquid Sloshing Dynamics: Theory and Applications (Cambridge University Press, Cambridge, England, 2005).

[6] O. M. Faltinsen and A. N. Timokha, Sloshing (Cambridge University Press, Cambridge, England, 2009).

[7] R. E. Hutton, An investigation of resonant, nonlinear, nonplanar free surface oscillations of a fluid, NASA Technical Report D-1870 (1963).

[8] J. W. Miles, Nonlinear surface waves in closed basins, J. Fluid Mech. 75, 419 (1976).

[9] J. W. Miles, Resonantly forced surface waves in a circular cylinder, J. Fluid Mech. 149, 15 (1984).

[10] M. Funakoshi and S. Inoue, Surface waves due to resonant horizontal oscillation, J. Fluid Mech. 192, 219 (1988).

[11] A. Royon-Lebeaud, E. J. Hopfinger, and A. Cartellier, Liquid sloshing and wave breaking in circular and square-base cylindrical containers, J. Fluid Mech. 577, 467 (2007).

[12] M. Reclari, M. Dreyer, S. Tissot, D. Obreschkow, F. M. Wurm, and M. Farhat, Surface wave dynamics in orbital shaken cylindrical containers, Phys. Fluids 26, 052104 (2014).

[13] J. Bouvard, W. Herreman, and F. Moisy, Mean mass transport in an orbitally shaken cylindrical container, Phys. Rev. Fluids 2, 084801 (2017).

[14] F. Moisy, J. Bouvard, and W. Herreman, Counter-rotation in an orbitally shaken glass of beer, Europhys. Lett. 122, 34002 (2018).

[15] O. M. Faltinsen and A. N. Timokha, An inviscid analysis of the Prandtl azimuthal mass transport during swirl-type sloshing, J. Fluid Mech. 865, 884 (2019).

[16] G. Lindgren, Some properties of a normal process near a local maximum, Ann. Math. Stat. 41, 1870 (1970).

[17] P. Boccotti, Some new results on statistical properties of wind waves, Appl. Ocean Res. 5, 134 (1983).

[18] P. S. Tromans, A. R. Anaturk, and P. Hagemeijer, A new model for the kinematics of large ocean wavesApplication as a design wave, in Proceedings of the 1st International Offshore and Polar Engineering Conference, Edinburgh, UK (International Society of Offshore and Polar Engineers, 1991).

[19] P. Jonathan and P. H. Taylor, On irregular, nonlinear waves in a spread sea, J. Offshore Mech. Arctic Eng. 119, 37 (1997).

[20] H. Santo, P. H. Taylor, R. Eatock Taylor, and Y. S. Choo, Average properties of the largest waves in Hurricane Camille, J. Offshore Mech. Arctic Eng. 135, 011602 (2013). 
[21] C. N. Whittaker, A. C. Raby, C. J. Fitzgerald, and P. H. Taylor, The average shape of large waves in the coastal zone, Coastal Eng. 114, 253 (2016).

[22] W. Zhao, H. A. Wolgamot, P. H. Taylor, and R. Eatock Taylor, Gap resonance and higher harmonics driven by focused transient wave groups, J. Fluid Mech. 812, 905 (2017).

[23] H. Santo, P. H. Taylor, A. H. Day, E. Nixon, and Y. S. Choo, Current blockage and extreme forces on a jacket model in focused wave groups with current, J. Fluids Struct. 78, 24 (2018).

[24] L. F. Chen, J. Zang, P. H. Taylor, L. Sun, G. C. J. Morgan, J. Grice, J. Orszaghova, and M. T. Ruiz, An experimental decomposition of nonlinear forces on a surface-piercing column: Stokes-type expansions of the force harmonics, J. Fluid Mech. 848, 42 (2018).

[25] W. Zhao, P. H. Taylor, H. A. Wolgamot, and R. Eatock Taylor, Identifying linear and nonlinear coupling between fluid sloshing in tanks, Roll of a barge and external free-surface waves, J. Fluid Mech. 844, 403 (2018).

[26] Y. L. Shao and O. M. Faltinsen, A harmonic polynomial cell (HPC) method for 3D Laplace equation with application in marine hydrodynamics, J. Comput. Phys. 274, 312 (2014).

[27] H. Liang, O. M. Faltinsen, and Y.-L. Shao, Application of a 2D harmonic polynomial cell (HPC) method to singular flows and lifting problems, Appl. Ocean Res. 53, 75 (2015).

[28] A. Bardazzi, C. Lugni, M. Antuono, G. Graziani, and O. M. Faltinsen, Generalized HPC method for the Poisson equation, J. Comput. Phys. 299, 630 (2015).

[29] S. Ma, F. C. W. Hanssen, M. A. Siddiqui, M. Greco, and O. M. Faltinsen, Local and global properties of the harmonic polynomial cell method: In-depth analysis in two dimensions, Int. J. Numer. Methods Eng. 113, 681 (2018).

[30] F. C. W. Hanssen, A. Bardazzi, C. Lugni, and M. Greco, Free-surface tracking in 2D with the harmonic polynomial cell method: Two alternative strategies, Int. J. Numer. Methods Eng. 113, 311 (2018).

[31] H. Yan and Y. Liu, An efficient high-order boundary element method for nonlinear wave-wave and wavebody interactions, J. Comput. Phys. 230, 402 (2011).

[32] O. M. Faltinsen, I. A. Lukovsky, and A. N. Timokha, Resonant sloshing in an upright annular tank, J. Fluid Mech. 804, 608 (2016).

[33] M. Abramowitz and I. A. Stegun, Handbook of Mathematical Functions: With Formulas, Graphs, and Mathematical Tables, 55 (National Bureau of Standards, Gaithersburg, MD, 1964).

[34] A. P. Engsig-Karup, H. B. Bingham, and O. Lindberg, An efficient flexible-order model for 3D nonlinear water waves, J. Comput. Phys. 228, 2100 (2009).

[35] J. Berland, C. Bogey, O. Marsden, and C. Bailly, High-order, low dispersive and low dissipative explicit schemes for multiple-scale and boundary problems, J. Comput. Phys. 224, 637 (2007).

[36] A. Savitzky and M. J. E. Golay, Smoothing and differentiation of data by simplified least squares procedures, Anal. Chem. 36, 1627 (1964).

[37] See Supplemental Material at http://link.aps.org/supplemental/10.1103/PhysRevFluids.5.084801 for periodically modulated swirling waves in clockwise and anticlockwise direction, and for time-harmonic swirling waves in clockwise and anticlockwise direction.

[38] J. N. Newman, Marine Hydrodynamics (MIT Press, Cambridge, MA, 1977).

[39] H. Santo, P. H. Taylor, E. C. Moreno, P. Stansby, R. Eatock Taylor, L. Sun, and J. Zang, Extreme motion and response statistics for survival of the three-float wave energy converter M4 in intermediate water depth, J. Fluid Mech. 813, 175 (2017). 